\title{
1 Nutritional dependence of sperm mitochondrial metabolism and 2 small RNA biogenesis
}

3 Rashmi Ramesh ${ }^{1 *}$, Signe Skog ${ }^{1}$, Daniel Nätt ${ }^{1}$, Unn Kugelberg ${ }^{1}$, Lovisa Örkenby ${ }^{1}$,

4 Anita Öst $^{1^{*}}$

$5 \quad{ }^{1}$ Department of Biomedical and Clinical Sciences, Linkoping University, 58183 Linkoping, Sweden

$6 \quad{ }^{*}$ Correspondence to rashmi.ramesh@liu.se and anita.ost@liu.se

7 Lead contact: anita.ost@liu.se

\section{Summary}

9 A wide spectrum of exogenous factors, including diet, environmental pollutants,

10 stress, and seasonal changes have major impact on sperm quality and function. The

11 molecular basis, however, that explains this susceptibility remains largely unknown.

12 Using a combination of proteomics and small RNA (sRNA) sequencing, we show that

13 Drosophila sperm display rapid molecular changes in response to dietary sugar, both in terms of metabolic/redox proteins and sRNA content, particularly miRNA and mitochondria derived sRNA (mt-sRNA). Thus, results from two independent omics point at the dynamics of mitochondria as the central aspect in rapid metabolic adjustments in sperm. Using specific stains and in vivo redox reporter flies, we show that diet indeed rapidly alters the production of mitochondrial derived reactive oxygen species (ROS). Quenching ROS via supplementation of $\mathrm{N}$ acetyl cysteine reduces diet-upregulated miRNA, but not mitochondrial-sRNA. Together, these results open new territories in our search for the mechanistic understanding of sperm health and disease.

\section{Keywords}

24 Diet, sperm, proteomics, small RNA, ROS, mitochondrial ROS, mitochondrial small RNA, miR-10, tsRNA

\section{Highlights}

- Diet rapidly changes the proteomic and sRNA profiles in sperm

- Diet sensitive sperm proteins are found in human infertility studies

- Sperm mitochondrial ROS levels are modulated by diet

- dme-miR-10 regulation is secondary to diet-induced ROS

- Diet, but not diet-induced ROS, alters the expression of mitochondrial small 


\section{Introduction}

The male germ cell, the harbinger of subsequent generation's genetic material is surprisingly sensitive to environmental perturbations. In addition to endogenous factors, environmental and lifestyle-related factors including metabolic disorders such as obesity and type II diabetes impacts sperm quality, causing male infertility (Cescon, Chianese, \& Tavares, 2020; Chianese et al., 2017; Day et al., 2016; Du Plessis et al., 2010; Katib, 2015; Liu \& Ding, 2017; Pergialiotis et al., 2016; Schagdarsurengin \& Steger, 2016; Tavares et al., 2016). Such sensitivity of the sperm has been assumed to be secondary to suboptimal spermatogenesis, and consequently, exploratory studies investigating links between environmental shifts and sperm function have employed long-term or chronic intervention. Recently, however, we and others, have found that interventions shorter than the duration of spermatogenesis modulate the molecular composition of the sperm (Gapp et al., 2021; Nätt et al., 2019; Trigg et al., 2021). An enticing and plausible explanation for such rapid responses is that exosomes produced in the epididymis transport material to the sperm during the maturation process (Sharma et al., 2018; Trigg et al., 2021).

Sperm-borne small RNA have been shown to be sensitive to environmental perturbations (Donkin et al., 2016; Fullston et al., 2016; Fullston et al., 2013; Hua et al., 2019; Nätt et al., 2019) and actively influence offspring phenotypes (Chen, Yan, Cao, et al., 2016; Gapp et al., 2014; Grandjean et al., 2015; Rassoulzadegan et al., 2006; Rodgers, Morgan, Leu, \& Bale, 2015; Sharma et al., 2016; Trigg et al., 2021). In parallel, links between sRNA and male infertility are becoming increasing clear, with sRNA being suggested as potential biomarkers for male reproductive health (Abu-Halima et al., 2014; Hua et al., 2019; B. Nixon et al., 2019; Salas-Huetos et al., 2016). This duality of sperm sRNA as a potential biomarker for infertility and their importance in early embryonic development, combined with our finding that some diet-sensitive sRNA correlates with sperm motility (Nätt et al., 2019), suggests a shared aetiology for male factor infertility and intergenerational metabolic responses (Nätt \& Öst, 2020).

The sperm mitochondria are important for optimal motility and full reproductive potential. Subsequently, mitochondrial function has been studied in relation to male infertility (recently review in Boguenet et al., 2021). For example, comparative 
proteomics of human sperm have pinpointed mitochondrial proteins to be downregulated in patients with athenozoospermia (Agarwal et al., 2016; Amaral et al., 2014; Bui et al., 2018; Moscatelli et al., 2019; Nowicka-Bauer et al., 2018). Additionally, mitochondria are primary ROS producers in the sperm (Koppers, et al., 2008; Kothari et al., 2010) and ROS acts as a double-edged sword-providing for basal physiologic function of sperm as well as creating oxidative damage. ROSproduction has been studied in the context of male infertility (Agarwal et al., 2019; Evans et al., 2021; MacLeod, 1943; Tremellen, 2008). Despite recent advances facilitated by the usage of high-throughput techniques, such as transcriptomics, metabolomics and proteomics providing insights into the basic molecular composition and mechanisms of spermatogenesis and male infertility (Carrell et al., 2016), little is known about the mechanisms connecting rapid responses to diet, molecular changes in sperm and ultimately male fertility. With the rise in global male infertility (Sengupta, Dutta, \& Krajewska-Kulak, 2017) there is a need for a comprehensive approach to longitudinally link paternal diet to changes observed in sperm.

Using our Drosophila model for paternal intergenerational metabolic response (IGMR) (Öst et al., 2014), we demonstrate that both proteomic and sRNA profiles in sperm are dynamic. We observe that certain diet-regulated proteins are known markers of male infertility, thus strengthening the link between diet and fertility. A short dietary intervention with varying amounts of sugar also resulted in rapid changes in the sperm mitochondrial ROS production. We show that miRNAespecially the upregulation of miR-10 is a result of diet-induced ROS. Next, we focused on tRNA derived fragments (tsRNA), which has been implicated in dietinduced intergenerational responses in many species (Chen et al., 2016; Nätt et al., 2019; Sharma et al., 2016). Finally, combining our highly controlled Drosophila model with human sperm sRNA data we identified mitochondrial tsRNA as major components in diet-dependent regulation of sperm across species.

\section{Results}

\section{A brief dietary intervention rapidly modifies the sperm proteome.}

We first explored the proteomic landscape of the sperm and sperm microenvironment using proteomics. Briefly, seminal vesicles with mature sperm were dissected for protein extraction and subjected to mass spectrometry (Figure 1A). In total, 542 
proteins were identified (Figure 1A- pie chart, Table S1), of which 149 proteins were also found in at least two previously published studies (Dorus et al., 2006; Takemori \& Yamamoto, 2009; Wasbrough et al., 2010) (Table S2). Moreover, 65 proteins in our dataset (Table S3), were also present in MitoDrome- a database for nuclear encoded-mitochondrial proteins in Drosophila melanogaster (Sardiello, et al., 2003; D'Elia et al., 2006). Importantly, the nine most abundant proteins in our dataset were all sperm-specific (Figure S1A), including sperm-exclusive proteins such as loopin-1 and $\beta$-Tubulin 85D (betaTub85D) (Bastian et al., 2021). Functionally, most of the identified proteins were involved in cellular metabolic pathways, while others belonged to the broad categories of translation and protein synthesis, heat-shock response, and accessory gland proteins (Figure 1A- pie chart, Table S1). The substantial coverage of our data with published studies, along with the identification of primarily metabolic proteins in sperm, justified further functional investigation in relation to dietary intervention.

Three groups of adult virgin male flies were, therefore, fed differing amounts of sugar (10-fold increases viz 3, 30 or $300 \mathrm{~g} / \mathrm{L}$ ) for two days (Figure 1A). A subset of proteins was differentially expressed (Figure 1B), but the most abundant sperm specific proteins - such as loopin-1 and betaTub85D - remained stable in all three dietary conditions (Figure S1B). Unsupervised hierarchical clustering of the differentially expressed proteins $(p<0.08)$ revealed two major clusters (Figure $1 \mathrm{~B}$, clusters $1 \& 2)$. Whilst proteins involved in TCA cycle (CS, Fum2, CG32026, Irp-1B) and pyruvate metabolism (PyK, Gapdh and Menl-2) were enriched in cluster 1, translation-related proteins were dominating cluster 2 (Rpl22-like, Rpl7, RpL8, elF4A, EF2) (Figure 1C). Strikingly, proteins in each category (viz TCA, pyruvate metabolism or redox) showed a similar trend of expression across the conditions tested (Figure 1B, $2 \mathrm{~A}$ and $2 \mathrm{E}$ ), indicating that these metabolic pathways are rapidly modulated by diet in sperm. To gain more translational and functional insights, we studied their human orthologues in silico. Interestingly, many of the proteins present in Figure 1B had previously been reported in human male infertility (CTD Gene-Disease Associations dataset) (Rouillard et al., 2016). Among these were well studied protein biomarkers for male infertility such as IDH3A, GAPDHS, FH, DLAT (Figure 1B, red text) (Agarwal et al., 2020; Torra-Massana et al., 2021). This translational overlap (Figure 1B) supports the hypothesis of a link between paternal diet (sugar) and infertility. We, 
131 therefore, tested the infertility phenotype in flies via RNAi mediated knockdown of

132 single candidate proteins in the germline (Figure S1C). Among the tested RNAi lines,

133 knockdown of elF4A, CCT4 and mitofilin resulted in strong phenotypes- with smaller

134 testes and a complete absence of mature sperm (representative micrographs for

135 elF4A knockdown in Figure S1D).

136 In summary, our findings highlight the rapid shift in the sperm proteome after a brief

137 dietary intervention. Notably, the human orthologues of the shifted proteome are

138 implicated in male infertility providing important clues about a mechanism associating

139 diet with male infertility.

140 Diet acutely modulates ROS production in the sperm.

141 Careful inspection of the differentially expressed proteins (Figure 1B) revealed a

142 subset of proteins that is involved in stress /redox homeostasis (Figure 2A). The

143 expression of these proteins, namely Gsts1, Nlaz, Gld, elF4A, TER94, glob1 and

144 PP1-13c, were higher in $30 \mathrm{~g} / \mathrm{L}$ compared to the other two diets (Figure 2A). We,

145 therefore, hypothesised that the flies eating $30 \mathrm{~g} / \mathrm{L}$ sugar would differ in sperm ROS

146 production compared to flies eating more or less sugar.

147 Seminal vesicles from flies with a sperm-specific GFP-reporter -Don juan GFP ( $D j$ -

148 GFP), that ate 3-, 30- or $300 \mathrm{~g} / \mathrm{L}$ sugar for two days, were dissected and incubated

149 with the ROS-indicator (CellROX® Orange). Visualization of the live tissues revealed

150 a striking difference in ROS production in response to diet (Figure 2B). Sperm from

$15130 \mathrm{~g} / \mathrm{L}$ sugar diet showed distinct orange fluorescence indicative of active ROS

152 production, while negligible/no fluorescence was detected in low- and high-sugar

153 diets (Figure 2B, C- upper panel). The differences in fluorescence were not a result of

154 changes in the amount of sperm per se, since GFP fluorescence corresponding to $D j-$

155 GFP from sperm tails remained unchanged (Figure 2B, 2C; lower panel). We also

156 independently replicated the findings in a separate fly strain $\left(\mathrm{W}^{1118}\right)$ using a general

157 ROS indicator dye ( $\mathrm{H}_{2}$ DCFDA) (Figure 2D). Together, this clearly suggests that a diet

158 comprising $30 \mathrm{~g} / \mathrm{L}$ sugar promotes ROS production in the sperm of Drosophila

159 melanogaster.

160 Proteins involved in TCA cycle were upregulated in seminal vesicles of $30 \mathrm{~g} / \mathrm{L}$ sugar-

161 eating males (Figure1B, 2E), whilst proteins involved in glycolysis were 
162 downregulated (Figure1B, 2E). Given that the TCA cycle operates in the

163 mitochondria, and that mitochondria are significant ROS producers in sperm

164 (Koppers et al., 2008; Kothari et al., 2010) we hypothesised that diet-induced ROS

165 originates in the mitochondria.

166 We, therefore, used a previously characterised ratiometric $\mathrm{H}_{2} \mathrm{O}_{2}$ redox sensor,

167 mitochondrial-roGFP2-Orp1 (Albrecht et al, 2011) (Figure S2A-C). This in vivo sensor

168 allowed us to specifically track mitochondrial ROS, with the added advantage of

169 defining $\mathrm{H}_{2} \mathrm{O}_{2}$ as the specific ROS species modulated by diet. As previously

170 described (Figure 1A), 3-5-day old male flies were fed 30- or $300 \mathrm{~g} / \mathrm{L}$ sugar for 2

171 days, and fluorescence was measured by confocal microscopy by sequential

172 excitation at 405- and $488 \mathrm{~nm}$ (See methods for details). In line with the TCA-related

173 protein changes, we found that $30 \mathrm{~g} / \mathrm{L}$ condition presented seminal vesicles with a

174 higher 405/488 ratio suggesting the involvement of the mitochondria in creating the

175 oxidative environment via $\mathrm{H}_{2} \mathrm{O}_{2}$ production (Figure $2 \mathrm{~F}$, Figure $\mathrm{S} 2 \mathrm{C}$ ).

176 In mitochondria, superoxide radicals are produced mainly from the electron transport

177 chain (ETC) and are converted to $\mathrm{H}_{2} \mathrm{O}_{2}$ by the enzyme superoxide dehydrogenase

178 (SOD) (Figure 2G). To validate that diet modulates the mitochondrial $\mathrm{H}_{2} \mathrm{O}_{2}$

179 production, we initiated a germline specific knockdown of the mitochondrial enzyme-

180 Sod1 (Figure S1C) and measured ROS levels by microscopy (as in Figure 2B, C). As

181 expected, quantification of fluorescence in seminal vesicles after RNAi revealed a

182 reduction in the amounts of mitochondrial ROS irrespective of diet, while the

183 genetically unmodified controls on a $30 \mathrm{~g} / \mathrm{L}$ sugar diet showed ROS generation

184 (Figure 2H). Together, these results highlight the prominent redox changes in sperm

185 mitochondria in response to diet.

186 We next turned to investigating whether mitochondrial function itself was modulated

187 by diet. Since $30 \mathrm{~g} / \mathrm{L}$ favoured ROS production in comparison to $300 \mathrm{~g} / \mathrm{L}$ sugar, we

188 reasoned that either mitochondrial morphology or activity was altered. We, therefore,

189 measured mitochondrial potential $\left(\Delta \Psi_{\mathrm{m}}\right)$. Seminal vesicles from flies eating 30 - or

$190300 \mathrm{~g} / \mathrm{L}$ sugar for two days were dissected and incubated with a fluorescent dye that

191 specifically stains the mitochondria (MitoTracker ${ }^{\mathrm{TM}}$ Red CMRos). The uptake of this

192 cell-permeable dye is dependent on $\Delta \Psi_{\mathrm{m}}$, whereby a strong signal of the dye

193 indicates a high mitochondrial potential. Bright red fluorescence was seen in seminal 
194 vesicles from flies feeding either 30 - or $300 \mathrm{~g} / \mathrm{L}$ sugar (Figure S2D). Quantification of

195 red fluorescence in seminal vesicles (containing mature sperm) revealed no

196 significant differences in sperm of either diet (Figure 2I). ROS levels in seminal

197 vesicles were measured like in Figure 2D on a separate set of flies from the same

198 experiment, and as observed before, $30 \mathrm{~g} / \mathrm{L}$ favoured ROS production (Figure S2E).

199 These results suggest that although diet had a rapid effect on ROS production in

200 sperm, there was little to no effect on the mitochondrial $\Delta \Psi_{\mathrm{m}}$ in mature sperm.

201 Although a general change in $\Delta \Psi_{\mathrm{m}}$ was not detected, it is possible that specific sites

202 in the ETC are affected by diet. Indeed, previous studies where individual ETC

203 complexes were modulated, support ROS-mediated signalling via both conventional

204 as well as reverse electron transport (reviewed in (Scialò, Fernández-Ayala, \& Sanz,

205 2017)). Thus, future studies on dissecting the role of individual complexes are

206 needed. What is clear, however, is that a dietary change of just two days is reflected

207 in the sperm mitochondrial $\mathrm{H}_{2} \mathrm{O}_{2}$ production.

\section{Acute dietary intervention rapidly modifies the sRNA profiles in sperm}

209 Having found that mitochondrial ROS respond rapidly to diet, we sought to explore

210 whether sperm sRNA profiles were similarly shifted, and if so, whether such changes

211 would be secondary to diet-induced ROS. For this, we took advantage of the direct

212 quenching of ROS with general antioxidants such as $\mathrm{N}$ acetyl cysteine (NAC)

213 supplemented in diet containing either 30 - or $300 \mathrm{~g} / \mathrm{L}$ sugar (Figure 3A). After two

214 days, the seminal vesicles were dissected and incubated with CellROX®, and ROS

215 was measured by microscopy and quantified (Figure 3A). As previously observed,

216 the $30 \mathrm{~g} / \mathrm{L}$ sugar diet resulted in high ROS production in sperm, while flies fed 300

$217 \mathrm{~g} / \mathrm{L}$ sugar had little to no ROS (Figure 3A, -NAC). NAC supplementation $(1 \mathrm{mg} / \mathrm{mL})$ in

218 both 30 - and $300 \mathrm{~g} / \mathrm{L}$ diets greatly diminished the ROS levels (Figure 3A, +NAC),

219 indicating the effectiveness of the antioxidant in quenching the diet-induced ROS in

220 seminal vesicles. We therefore used NAC to investigate the effect of diet-induced

221 ROS on sperm sRNA. Importantly, to eliminate the contribution from somatic sRNA,

222 only pure mature sperm was used for sRNA sequencing (Figure 3A, B).

223 Sperm sRNA sequencing revealed the presence of different sRNA biotypes including,

224 in order of their abundance, rRNA-derived small RNA (rsRNA), tRNA-derived small

225 RNA (tsRNA), mitochondrial tRNA-derived small RNA (mt tsRNA), microRNA

226 (miRNA), long intergenic non-coding RNA (lincRNA), piwi-interacting RNAs (piRNA) 
and sRNA from protein- coding RNA (Table S4). For comparison, we analysed human sperm sRNA data from Nätt et al. 2019. Reads mapping to rsRNA dominate the sperm of both flies and human (94\% versus $74 \%$ respectively, see Table S4 for rsRNA information in fly sperm). We did not detect gross changes in the nuclear rsRNA profiles and have therefore excluded them from our analyses due to their high presence.

A side-by-side comparison of sRNA from flies and human sperm (Nätt et al. 2019) revealed striking similarities in the distribution and abundance of various sRNA biotypes, with tsRNA being the abundant biotype in sperm of both species (Figure 3B), as previously reported in mature sperm in mice (Peng et al., 2012). The abundance of the other sRNA biotypes varied more between fly and human sperm. Notably, compared to human, miRNA and piRNA had a stronger presence in fly sperm (Figure 3B). Nonetheless, global similarities of sRNA biotypes between fly and human justifies the use of a fly model to better understand mechanisms in human sperm.

242 Next, we looked at the size distribution of various sRNA biotypes in sperm of flies fed 243 with 30- and $300 \mathrm{~g} / \mathrm{L}$ sugar with and without NAC (Figure $3 \mathrm{C}$ ). The size distribution 244 showed diverse lengths of tsRNA, spanning 18-50 nucleotides (Figure 3C). Notably, the proportions of the various biotypes in the range of 18-30 nucleotides remained similar across the four conditions, suggesting that diet or NAC did not induce a major shift in the profiles of these sRNA (Figure 3C). The fold change distribution of sRNA biotypes, however, revealed more specific effects between conditions (Figure 3D). Whilst tsRNA showed a diverse spread across the conditions (Figure 3D, closed blue 250 circles), miRNA were predominantly upregulated in $30 \mathrm{~g} / \mathrm{L}$ condition (Figure 3D, quadrant II, and Figure S3A, B). In parallel, mt-tsRNA were downregulated in the 30 g/L condition (Figure 3D, closed yellow circles, quadrant IV, and Figure S3A). upregulation of several miRNAs (Nätt et al., 2019). In the current study, almost all miRNA were upregulated in $30 \mathrm{~g} / \mathrm{L}$ diet both in the presence and absence of NAC.

256 Intrigued by the finding that most miRNAs (84.8\% and $98.3 \%$ respectively, Figure S3 257 A, B \& Table S5) were upregulated, we decided to further investigate specific 258 miRNAs. Interestingly, the most differentially expressed miRNA -dme-miR-10 (CPM 
of $30 \mathrm{~g} / \mathrm{L}$ vs $300 \mathrm{~g} / \mathrm{L}$ ) reached clear statistical significance in the $30 \mathrm{~g} / \mathrm{L}$ condition $(p<0.05$, Figure 3E asterisk *, Figure 3F). It has been shown that the rodent and human orthologue, miR-10, is diet responsive (Cropley et al., 2016), and is upregulated in male infertility of both rodents and human (Gao et al., 2019). Among the upregulated miRNA (Figure 3E \& Figure S3C), is miR-276b, which is a known regulator of synchronous egg-hatching in locusts (He et al., 2016), suggesting that sperm miRNA may have long-lasting effects in early embryogenesis. Interestingly, in the presence of NAC, miR-10 is downregulated (Figure 3F, miR-10). Both the 5' and 3' arms of miR-10, called miR-10a and miR-10b respectively, were upregulated in the $30 \mathrm{~g} / \mathrm{L}$ sperm and similarly quenched by NAC (Figure 3G), making miR-10 a strong candidate for future research. The upregulation of miRNA in the ROS-producing diet (30 $\mathrm{g} / \mathrm{L}$ sugar), and the mitigation of this effect by NAC supports the idea that miRNA regulation is secondary to the diet-induced ROS production in sperm.

\section{Sperm tsRNA show varied responses to diet and ROS}

We next analysed the tsRNAs in both $30-$ and $300 \mathrm{~g} / \mathrm{L}$ sugar diets with and without NAC (Figure 4, Table S6). First, all transcripts mapping to full-length tRNAs were classified based on cut-sites using the bioinformatic package-Seqpac (Skog et al., 2021) and with information about tRNA loop structure taken from tRNAscan-SE (Lowe \& Chan, 2016). Five sub-types of tsRNA were defined: 5'-half, 5'-tsRNA, i'tsRNA, 3'-tsRNA and 3'-half (Figure 4A). The distributions of the tsRNA subtypes varied depending on whether they were of nuclear or mitochondrial origin (Figure 4B), suggesting alternative pathways of biogenesis. The nuclear tsRNA were dominated by 5'-halves (Figure 4B-nuclear tsRNA, \& Figure S4A), as reported previously (Chen et al., 2016; Nätt et al., 2019; Sharma et al., 2016). On the other hand, i'-tsRNA were predominant amongst the mitochondrial tsRNA (Figure 4Bmitochondrial tsRNA). This indicates distinct cleavage signatures in the two subcellular compartments. More importantly, the tRNA cleavage sites were mainly dependent on the transcriptional origin of the tRNA rather than the diet itself (Figure S4B, C).

The observed downregulation of mt-tsRNA in the $30 \mathrm{~g} / \mathrm{L}$ condition (Figure 3D), and the tsRNA cleavage signature in the mitochondria (Figure 4B) raised an intriguing possibility that tsRNA biogenesis in the mitochondria might be a direct consequence 
291 of diet-mediated responses. Therefore, to get an overview on ROS mediated tRNA

292 cleavage, all fragments carrying the same anticodon sequence (isodecoders and

293 isoacceptors) were combined and mean CPM values were visualized as a heatmap

294 (Figure 4C). Unsupervised clustering of these tsRNA revealed four clusters (Figure

2954 C, clusters 1-4). Interestingly, the mitochondrial tsRNA (Figure 4C, highlighted in

296 blue text) and nuclear tsRNA (Figure 4C, black text) formed separate clusters, as

297 previously observed in human sperm (Nätt et al., 2019), which again indicates

298 different biogenesis pathways. The mitochondrial tsRNA were generally less

299 abundant in $30 \mathrm{~g} / \mathrm{L}$ compared to $300 \mathrm{~g} / \mathrm{L}$ diet (Figure 4C, 30- \& $300 \mathrm{~g} / \mathrm{L}$, -NAC). This

300 downregulation of some mitochondrial tsRNA in $30 \mathrm{~g} / \mathrm{L}$, namely lle-GAT, Gly-TCC

301 and Met-CAT was statistically significant (Figure 4C, asterisks* \& Figure 4D). Most

302 importantly, the presence of NAC did not alter the diet-induced shift of mitochondrial

303 tsRNA (Figure 4C, 30- \& $300 \mathrm{~g} / \mathrm{L},+\mathrm{NAC}$ ). This supports the hypothesis that changes

304 in mt-tsRNA happens prior to diet-induced mitochondrial ROS.

305 The nuclear pool of tsRNA (Figure 4C, clusters 2-4) included fragments previously

306 identified as being diet-sensitive (Figure 4C, see \#) (Chen et al., 2016; Sharma et al., 307 2016). Notably, three sub-clusters emerged among the nuclear tsRNA (Figure 4C,

308 clusters 2-4). TsRNA in cluster 3 had a higher presence in both diets (Figure 4C, 30-

$309 \& 300 \mathrm{~g} / \mathrm{L},-\mathrm{NAC})$, while addition of NAC reduced the levels of the same tsRNA

310 (Figure 4C, 30- \& $300 \mathrm{~g} / \mathrm{L},+\mathrm{NAC}$ ), indicating alternative sources of tRNA

311 fragmentation in the presence of NAC. It is plausible that non-diet induced ROS,

312 could have been quenched by the presence of NAC, giving rise to these fragments.

313 The nuclear tsRNAs in cluster 4 show indistinct effects of both diet and NAC (Figure

314 4C, cluster 4). This suggests that multiple mechanisms are at play and ROS or diet,

315 alone or in combination exert multiple effects on certain tsRNA.

316 Together, these findings point at dynamic changes in the tsRNA profiles in sperm

317 mitochondria in response to diet, further highlighting the role of mitochondria in diet-

318 induced metabolic alterations in sperm. In fact, comparing diet-sensitive mt-tsRNA in

319 humans (Nätt et al., 2019) and flies shows striking similarities, with at least $50 \%$ of

320 the observed mt-tsRNA being altered by diet (Figure 4E). 


\section{Discussion}

323

324

Here, we have used proteomics and sRNA sequencing, combined with in-depth bioinformatic analyses in the Drosophila model of paternal intergenerational metabolic response (IGMR), to identify the molecular changes in the sperm. A short dietary intervention with varying amounts of sugar resulted in a rapid remodelling of the proteome in seminal vesicles containing mature sperm. Whereas the bulk of structural proteins was unaffected by diet (Figure S1B), we found changes in proteins involved in metabolism and stress /redox homeostasis (Figure 1A, B \& Figure 2A).

\section{This change in redox homeostasis was reflected in ROS production in sperm} mitochondria, which dramatically increased with $30 \mathrm{~g} / \mathrm{L}$ sugar (Figure $2 \mathrm{~B}-\mathrm{D}, \mathrm{F}$, \& Figure S2C). In parallel, high-throughput sequencing of purified sperm RNA revealed coordinated changes in the sperm sRNA. In depth analyses of the sRNA profiles indicated that response to diet in sperm manifested in different ways. NAC mediated depletion of ROS in sperm was able to reverse the expression of diet-altered miRNA, in particular miR-10 (Figure 3D-E), suggesting this change to be secondary to ROS. The nuclear and mitochondrial tRNA fragments (tsRNA) were also altered and separated into unique clusters suggesting a mixed response to diet and ROS (Figure 4C). More specifically, and in line with findings in human sperm, we found that diet, but not diet-induced ROS, altered the expression of the mt-tsRNAs (Figure 4C, D \& Figure S4B, C). Together, our results favour a model where mitochondrial metabolic flexibility and sRNA biogenesis are in the centre of diet-dependent molecular changes in sperm.

Since sperm rely on substrates from their microenvironment to fuel their metabolism, it is easy to envision that temporal changes in nutrient flux are directly mirrored in the sperm metabolism. Indeed, the existence of a gut-gonad axis has been demonstrated in Drosophila, wherein the male intestine secretes citrate to the adjacent testes and promotes sperm maturation (Hudry et al., 2019). A similar gutgonad axis was recently described in a sheep model of diet-induced metabolic syndromes (Zhang et al., 2021). In both these models, metabolic perturbations altered spermatogenesis and sperm numbers. However, with our short dietary intervention, two findings indicate unchanged sperm numbers: (1) highly expressed sperm proteins such as loopin-1 and beta-tubulin are maintained at the same level in 
354 the proteomics data independent of diet (Figure S1A), and (2) microscopy of seminal vesicles of the sperm-specific fusion protein Dj-GFP revealed no change in fluorescence intensity (Figure 2C). In addition, staining of seminal vesicles with MitoTracker ${ }^{\mathrm{TM}}$ Red CMXRos showed similar staining patterns across the tested diets, revealing negligible to no changes in sperm numbers or in mitochondria potential. It is, however, possible that long term dietary changes, or other nutrient compositions, would impede spermatogenesis and modulate the number of sperm being produced.

While sperm numbers appeared unchanged by diet, we observed that diet altered the mitochondrial $\mathrm{H}_{2} \mathrm{O}_{2}$ production. Given that $\mathrm{ROS}$ is required for sperm functions in 363 humans (Du Plessis et al., 2015; Dutta et al., 2020), modulation of such essential processes by diet is intriguing and can have far-reaching consequences. Given its easy diffusibility across membranes, and a longer half-life, $\mathrm{H}_{2} \mathrm{O}_{2}$ is considered one of the main signalling molecules amongst the ROS species (Holmström \& Finkel, 2014; Rhee, 2006; Veal, Day, \& Morgan, 2007). On the other hand, ROS-induced oxidative stress can lead to male infertility (Barati, Nikzad, \& Karimian, 2020;Agarwal, Makker, \& Sharma, 2008; Agarwal et al., 2019; Bui et al., 2018; Tremellen, 2008). Therefore, production of ROS must be counterbalanced by antioxidant systems. In mammals, it is well known that proteins secreted from the epididymis provide such a protection for the maturing sperm (reviewed in Chianese \& Pierantoni, 2021). Similarly, we find a rapid upregulation of certain enzymes involved in redox homeostasis - such as Gsts1 and Glob1 in the high ROS condition (Figure 2A). Considering the transcriptional and translational quiescence of the sperm, it is likely that somatic cells in the seminal vesicle has a similar role as the epididymis in providing an antioxidant environment for the sperm. In mammals, proteins (Candenas \& Chianese, 2020; Nixon et al., 2019) and sRNA made in somatic cells are known to be packaged and transported to sperm via extracellular vesicles (Belleannée et al., 2013; Sharma et al., 2016; Vojtech et al., 2014; Xu et al., 2020). For example, miR-10 expression have been shown high in seminal exosome in humans (Barceló et al., 2018; Vojtech et al., 2014), and in mice tsRNA, especially the tsRNA 5'-halves, are known to be loaded

383 onto the sperm via exosomes originating from the epididymis (Sharma et al., 2016;

384 Stanger et al., 2020). Although no changes in the abundance of tsRNA 5'-halves were detected in this study, the markers of extracellular vesicles such as Ter94, APOD, GAPDH (Figure 1B) and miR-10 (Figure 3F), were altered with diet, 
suggesting the involvement of exosomes in creating an antioxidant response in Drosophila seminal vesicle.

Alongside the upregulation of miRNA and proteins involved in stress /redox homeostasis interpreted as part of an antioxidant response to increased ROS, our data point to a separate mechanism for the biogenesis of mt-sRNA detected in sperm (Figure S3A, B). In general, very little is known about sRNA originating from the mitochondria, both regarding their biogenesis and their functionality. It has been reported that mitochondria sRNA, in particular mt-piRNA, is abundant in male mice germ cells (Larriba et al., 2018) . It is interesting to note that we observed about $92 \%$ of mt-piRNA to be downregulated in the $30 \mathrm{~g} / \mathrm{L}$ condition (Figure S3A) but we have yet to explore if they have a functional role in sperm and/or in the fertilized egg. Likewise, it remains an open question whether mt-tsRNA carries a similar regulatory function as has been demonstrated with nuclear tsRNA. Like nuclear tsRNA, the mitochondrial tsRNA in fly (Figure 4D) and human (Nätt et al., 2019) sperm have distinct cut-sites, indicating that sperm mt-tsRNA have specific functions. The role of tsRNA in relaying the paternal nutritional status to the offspring has been shown in rodent models (Chen et al., 2016; Sharma et al., 2016). Thus, it is tempting to speculate that mt-tsRNA have similar roles. Nonetheless, we show that cleavage of mt-tsRNA differs from that of nuclear tsRNA (Figure 4B). This supports recent data showing that the biogenesis of nuclear and mitochondrial tsRNAs differs in flies (Molla-Herman et al., 2020).

Sperm sRNA profiles, especially alterations in miRNA have been studied in infertile men (Abu-Halima et al., 2014; Lian et al., 2009; Muñoz, Mata, Bassas, \& Larriba, 2015; Salas-Huetos et al., 2016b; C. Wang et al., 2011), and have been suggested as biomarkers of male infertility (Kotaja, 2014; Barbu et al., 2021; Kiani, Salehi, \& Mogheiseh, 2019; Salas-Huetos et al., 2020). Additionally, sperm tsRNA, miRNA and rsRNA has recently been shown to correlate with embryo quality (Grosso et al., 2021; Hua et al., 2019; Nätt \& Öst, 2020; Xu et al., 2020) and piRNA are involved in paternal diet induced intergenerational response (Lempradl et al., 2021). We have earlier described that a short high-sugar intervention in healthy young men synchronously increases tsRNA and rsRNA coming from the mitochondrial genome and that the increase of mt-tsRNA is positively associated with simultaneous changes in sperm motility (Nätt et al., 2019). Since mitochondrial energy metabolism is 
420 intimately linked with motility, this is an intriguing finding. Moreover, reanalysing data

421 from Donkin et. al we observed that obesity is associated with less rsRNA derived

422 from mitochondrial DNA (Donkin et al., 2016; Nätt et al., 2019). In all, our data adds

423 to the findings that SRNA in sperm can be a good biomarker of male reproductive

424 health (Abu-Halima et al., 2014; Hua et al., 2019; B. Nixon et al., 2019; Salas-Huetos

425 et al., 2016) and provides evidence that both the nutrient state as well as the ROS-

426 production of the sperm can influence sperm health.

427 We conclude that Drosophila sperm are susceptible to dietary changes and have 428 identified candidates that could influence metabolic responses in offspring. With the

429 rise in male factor infertility identification of such biomarkers in sperm should

430 therefore be of prime interest in future investigation.

\section{Acknowledgements}

432 This research was supported by grants from The Swedish Research Council (2015-

433 03141), Ragnar Söderberg's foundation and, Knut and Alice Wallenberg foundation

434 (2015.0165) to A.Ö. Technical support from staff at core facilities at Linköping

435 university, especially Vesa Loitto (microscopy unit), Annette Molbaek and Åsa

436 Schippert (RNA sequencing) and Maria Turkina (mass spectrometry) is gratefully

437 acknowledged. The authors are grateful to Marie Roth for technical help with

438 dissections, and Anna Asratian and Cecilia Jönsson for comments on the manuscript.

\section{Author contributions}

440 Conceptualization: A.Ö. and R.R.; Methodology: R.R, S.S, U.K, D.N, L.Ö, A.Ö. Data

441 curation: S.S. Formal analysis, R.R, S.S, D.N, A.Ö., Resources: A.Ö, Software: S.S,

442 D.N., Visualization: R.R, S.S, D.N, A.Ö, Writing - original draft: R.R, S.S, A.Ö,

443 Writing - review \& editing: R.R, S.S, U.K, D.N, L.Ö, A.Ö

444 Funding acquisition: A.Ö Supervision

445 All authors have approved the final version of this paper.

\section{Declaration of interests}

447 The authors declare no competing interests. 
$449 \quad$ Figure legends

450 Figure 1: Rapid response to dietary sugar involves proteomic shifts in sperm

451 A: Schematics and Functional distribution of sperm proteome. 2- 3-day old

452 virgin $W^{1118}$ males were fed a diet containing 3-, 30- or $300 \mathrm{~g} / \mathrm{L}$ sugar for 2 days.

453 Seminal vesicles were dissected out and proteomics was performed by mass

454 spectrometry. Pie chart reveals the proteomic distribution. Metabolic proteins

455 constitute most of the proteome (in black), followed by proteins in other categories (in 456 colour).

457 B: Heatmap of significantly changed proteins $(p<0.08)$. Fly proteins and their human 458 orthologues are shown under their respective cartoon pictograms. $N=3$, with $n=20$ per 459 replicate, Pearsons distance based hierarchical clustering with complete linkage-

460 based clustering. Clusters 1 and 2 are indicated with grey bars on the right.

461 Significance test was performed with a linear model fit and Benjamini Hochberg 462 adjustment using $R$ package limma version 3.42.2. All comparisons are made to 30 $463 \mathrm{~g} / \mathrm{L}$ condition. Protein biomarkers of human male infertility are written in red text.

464 C: GO term and statistical overrepresentation analyses of proteins in clusters 1 and 2 465 using pantherdb. $\log _{2}$ Fold change calculated based on the entire fly genome is on $x$ 466 axis, and GO terms are in y axis. Coloured bars indicate the highest fold changes.

467 False discovery rate ( $p$ value) is given for each GO term.

468 See also Figure S1

469 Figure 2: Paternal diet rapidly changes mitochondrial ROS production in 470 sperm

471 A: Proteins involved in stress/ redox homeostasis found in Figure 1B are plotted 472 separately to indicate expression trends across the three diets. Mean $\log _{2}$ levels 473 (total counts) are on y axis, the different sugar concentrations are indicated on $x$ 474 axis.

475 B: Visualisation of ROS in seminal vesicle. Flies were fed different diets as in Figure $4761 \mathrm{~A}$ and their seminal vesicles were incubated with CellRox® dye. Images represent 477 ROS in mature sperm in orange channel (CellRox® orange) and Dj-GFP (sperm tail) 478 in green channel. ROS is seen clearly in $30 \mathrm{~g} / \mathrm{L}$ (middle panel), with minimal to no 479 orange fluorescence seen in $3-$ or $300 \mathrm{~g} / \mathrm{L}$ (top and bottom panels). $\mathrm{n}=4-6$, scale bar= $48050 \mu \mathrm{m}$. 
481 C: Quantification of ROS labelled with CellROX®. Seminal vesicles from B were 482 quantified using Fiji. CellRox® and GFP channels were separately quantified. Orange 483 bars represent CellRox®, and green bars represent the corresponding GFP

484 quantification. Asterisks $\left({ }^{*}\right)$ represent $p \leq 0.001$, unpaired t-test.

485 D: Quantification of ROS labelled with $\mathrm{H}_{2}$ DCFDA. Flies were fed different diets as in

486 Figure $1 \mathrm{~A}$ and seminal vesicles were incubated with a different ROS labelling dye-

$487 \mathrm{H}_{2}$ DCFDA. Fluorescence was quantified using Fiji. $\mathrm{n}=58-60$, asterisks $\left({ }^{*}\right)$ represent $p$ $488 \leq 0.0001$, unpaired t-test.

489 E: Proteins involved in pyruvate metabolism and tricarboxylic acid cycle (TCA) found 490 in Figure 1B are plotted separately to indicate expression trends across the three 491 diets.

492 F: Quantification of redox changes in mitochondrial $\mathrm{H}_{2} \mathrm{O}_{2}$ in seminal vesicles of flies 493 expressing the mito-roGFP2-orp1 ratiometric sensor. Ratio of fluorescence between 494405 and $488 \mathrm{~nm}$ wavelengths are plotted on y axis, and diets are indicated on $\mathrm{x}$ axis. $495 \mathrm{n}=20$, asterisks $\left({ }^{*}\right)$ denote $p \leq 0.05$. unpaired t-test.

496 G: Cartoon representation of sperm mitochondria, with the electron transport chain 497 (ETC) zoomed in (dotted box). Complexes I, II and III of the ETC are highlighted, and 498 superoxide production from oxygen is indicated $\left(\mathrm{O}_{2}^{--}\right)$in the intermembrane space.

499 Sod1 and catalase enzymes present in the mitochondrial intermembrane space are 500 shown to catalyse sequential reactions converting $\mathrm{O}_{2} \cdot-$ to $\mathrm{H}_{2} \mathrm{O}_{2}$, and $\mathrm{H}_{2} \mathrm{O}$ 501 respectively.

$502 \mathrm{H}$ : Quantification of ROS levels in wildtype flies and in flies with germline specific 503 knockdown via RNAi of Sod1 (Sod1-i). $n=4-5$ (Sod1-i) and n=18-24 (Wildtype), 504 unpaired t-test.

505 I: Quantification of mitochondrial potential $\left(\Delta \Psi_{\mathrm{m}}\right)$. Fluorescence intensities from

506 seminal vesicles stained with Mitotracker ${ }^{\mathrm{TM}}$ CMXRos. $\mathrm{n}=15$, unpaired t-test.

507 Note:

508 All comparisons were made to $30 \mathrm{~g} / \mathrm{L}$ condition (except $\mathrm{A}$ and $\mathrm{E}$ ).

509 The data shown are mean \pm SEM.

510 All quantification are done using Fiji (in $\mathrm{C}, \mathrm{D}, \mathrm{F}, \mathrm{H}, \mathrm{I}$ ) and mean grey values are plotted

511 on $y$-axis and diet is indicated on $x$ axis (except $F$ ).

512 a.u=arbitrary units, ns=no significant

513 See also Figure S2 
514 Figure 3: Rapid alteration in sperm sRNA following dietary intervention

515 A: Addition of antioxidant N-Acetyl Cysteine (NAC) mitigates ROS levels in sperm.

516 Following a 2-day dietary intervention, seminal vesicles were dissected out and ROS

517 levels were visualised using $\mathrm{H}_{2}$ DCFDA and quantified. The data shown are mean \pm

518 SEM. On a separate set of flies from the same experiment, sperm was isolated and

519 sequenced for sRNA.

520 B: Comparison of sRNA in fly and human sperm. Human sperm sRNA data were

521 derived from (Nätt et al., 2019). Shown in pie charts are percent CPM of perfect

522 matches to respective reference genome. Reads mapping to rsRNA are not shown.

523 Reads mapping to snoRNA (less than $2 \%$ ), snRNA (less than 1\%), and reads with no

524 annotation to any of the sRNA-subtype (less than 1\%) are classified as "others". Fly

525 data $n=20$, human data $n=15$.

526 C: Size distribution of sRNA in Drosophila sperm in the diets with and without NAC.

527 Reads mapping to rsRNA are excluded. Reads mapping to snoRNA, snRNA and

528 those sRNA that map to the reference genome but not to any other sRNA-subtypes

529 are consolidated as "other".

530 D: Scatter plot of fold changes showing the distribution of sRNA in the tested

531 conditions. $x$ axis represents $\log _{2}$ fold change (30 g/L+NAC) / (30 g/L) and y axis

532 represents $\mathrm{Log}_{2}$ fold change $(30 \mathrm{~g} / \mathrm{L}) /(300 \mathrm{~g} / \mathrm{L})$. Log fold changes was calculated on

533 CPM. Each point represents one individual sequence, in total 983. Coloured closed

534 circles are shown for miRNA (orange), mitochondrial tRNA (yellow), tRNA (blue) and

535 others (grey). Reads mapping to rsRNA are not included.

536 E: miRNA identified in this study. Fly miRNA are indicated under the fly pictogram.

537 Bars represent mean CPM. The corresponding closed circles represent mean

$538 \log _{2}$ fold change. Error bars are \pm SE. Human orthologues, where present, are

539 indicated under the human pictogram. Asterisks $\left(^{*}\right)$ indicate significance $(p<0.05)$

540 calculated by a Generalized Linear Negative Binomial model for all sequences that

541 originate from the same miRNA. NA= Not Available.

542 F: Bar graph for CPM expression of dme-miR-10 in all four dietary groups. Each point

543 represents the mean value of the two miRNA sequences mapping to dme-miR-10 for

544 each sample.

545 G: Coverage plot of dme-miR-10 showing expression of both the 5' (dme-miR-10a)

546 and 3' arms (dme-miR-10b). Each coloured line represents mean CPM of indicated

547 dietary condition. 
$548 \quad \mathrm{CPM}=$ counts per million

549 See also Figure S3

550 Figure 4: Sperm tsRNA have varied responses to diet and ROS

551 A: Illustrative representations of the fragments derived from tRNA analysed in this

552 study. See Methods section for details on tsRNA classification

553 B: Distribution of tsRNA based on their mapping to nuclear and mitochondrial

554 genomes. For each tsRNA, percentage of mean CPM is presented.

555 C: Heatmap showing expression of tsRNA. TsRNAs that originate from the same

556 mature tRNA are combined. Each column represents the mean CPM expression from

557 the indicated dietary condition. Clusters are based on unsupervised clustering and

558 are numbered 1-4 in red. The ' $m t^{\prime}$ in cluster 1 indicates tsRNA of mitochondrial origin.

559 Asterisk $\left({ }^{*}\right)$ represents statistical significance $(p<0.05)$ as determined by Negative

560 Binomial Generalized Linear Model. Hashtag (\#) refers to tsRNA identified in other

561 studies (Chen et al., 2016, Sharma et al., 2016).

562 D: Coverage plots of significantly altered mitochondrial tsRNAs (from C), namely Gly-

563 TCC, Ile-GAT and Met-CAT. $x$ axis shows the corresponding mature tRNA sequence,

564 and $y$ axis represents the mean CPM values.

565 E: Dietary regulation of mitochondrial tsRNAs is conserved from fly to man. Mt-

566 tsRNA are represented in yellow plain pies, with longitudinal black lined yellow pies

567 representing changes reaching statistically significance. Blue pies represent the

568 nuclear tsRNAs. In both flies (this study) and humans (Nätt et al., 2019), half of all

569 reported mitochondrial tsRNAs were significantly different with

570 sugar diet. Statistical significance $(p<0.05)$ was determined by Negative Binomial

571 Generalized Linear Model.

572 See also Figure S4 


\section{Materials and Methods}

\section{Fly stock maintenance and sugar diet administration}

576 A standard laboratory strain $\mathrm{W}^{1118}$ was used for proteomics, sRNA sequencing and certain microscopic experiments in this study. Dj-GFP males were used for ROS experiments to visualize sperm tail in seminal vesicles. The flies were inbred for several generations and maintained on standard cornmeal/molasses media at $26^{\circ} \mathrm{C}$. Male flies were isolated within 2 days of eclosure and aged for an additional 2-3 days before switching them to paternal diet intervention food containing 3,30 or $300 \mathrm{~g} / \mathrm{L}$

582 white sugar, for two days.

583 Standard food: Agar $10 \mathrm{~g} / \mathrm{l}$, yeast $28 \mathrm{~g} / \mathrm{l}$, cornmeal $68 \mathrm{~g} / \mathrm{l}$, molasses $68 \mathrm{~g} / \mathrm{l}$, Nipagin $5841.5 \mathrm{~g} / \mathrm{l}$, propionic acid $5.5 \mathrm{ml} / \mathrm{l}$.

585 Paternal diet intervention food: Agar $12 \mathrm{~g} / \mathrm{l}$, yeast $10 \mathrm{~g} / \mathrm{l}$, propionic acid $4,5 \mathrm{ml} / \mathrm{l}$, soy 586 flour $30 \mathrm{~g} / \mathrm{l}$ and white sugar as indicated.

\section{Isolation of seminal vesicles and preparation of protein extracts}

588 Seminal vesicles from 20 males for each diet were isolated in insect medium (Sigma 589 \# T3160) using fine forceps and collected in $25 \mu \mathrm{L}$ ice cold mili $\mathrm{Q}$ water in a $1.5 \mathrm{~mL}$ 590 microfuge tube stored on ice. After dissections of the complete set, tissue was lysed 591 mechanically using a fitted pestle (VWR \#431-0094), followed by 2 minutes (40 592 oscillation per second) on a bead shaker (Qiagen Tissue lyser)- centrifuged at $1000 \mathrm{~g}$ 593 for 5 minutes at $4{ }^{\circ} \mathrm{C}$ to remove debris, and supernatant was used for further 594 processing. $20 \mu \mathrm{L}$ of the supernatant was first alkylated in the presence of $10 \mathrm{mM}$ 595 DTT in ammonium bicarbonate $(25 \mathrm{mM})$ for 1 hour at $56^{\circ} \mathrm{C}$. Alkylation of cysteines 596 was performed using 55mM iodoacetamide prepared in ammonium bicarbonate 597 solution ( $25 \mathrm{mM}$ ) for 1 hour at room temperature in the dark. Following this, proteins 598 were precipitated using ice cold acetone overnight at $-20^{\circ} \mathrm{C}$. Subsequently 599 centrifuged at $15000 \mathrm{~g}$ for 10 minutes in a cooled rotor, and supernatant was 600 aspirated out. The pellet was used for Trypsin digestion (0.005 $\mu \mathrm{g} / \mu \mathrm{L}$, Pierce \#90057) 601 at $37^{\circ} \mathrm{C}$ overnight. The next morning, after a further boost of trypsin $(0.0025 \mu \mathrm{g} / \mu \mathrm{L})$ 602 for 3 hours at $37^{\circ} \mathrm{C}$, the digested peptides were vacuum-dried, and stored at $-2^{\circ} \mathrm{C}$. 603 Peptides were resuspended in $12 \mu \mathrm{L} 0.1 \%$ formic acid and used in duplicate $(5 \mu \mathrm{L})$ 604 for mass-spectrometry analysis. 


\section{Mass spectrometry analysis}

606 The peptides were introduced into an LTQ Orbitrap (Thermo, San Jose, CA) mass 607 spectrometer and all MS/MS samples were analysed using Sequest (Thermo Fisher 608 Scientific, San Jose, CA, USA; version IseNode in Proteome Discoverer 1.4.0.288). 609 Sequest was set up to search Fly uniprot 7227.fasta assuming the digestion enzyme 610 trypsin, with the following parameter settings: 1 miscleavages, variable methionine 611 oxidation and phosphorylation on serine and threonine, carboxymethyl cysteine as 612 fixed modification, with a fragment ion mass tolerance of $0.50 \mathrm{Da}$ and a parent ion 613 tolerance of 6.0 PPM. Results were merged using Scaffold (Proteome Software) 614 version 3.00.04.

\section{Criteria for protein identification}

616 Scaffold (version Scaffold_4.10.0, Proteome Software Inc., Portland, OR) was used 617 to validate MS/MS based peptide and protein identifications. Peptide identifications 618 were accepted if they could be established at greater than 95,0\% probability by the 619 Scaffold Local FDR algorithm. Protein identifications were accepted if they could be 620 established at greater than 99,0\% probability and contained at least 2 identified 621 peptides. Protein probabilities were assigned by the Protein Prophet algorithm 622 (Nesvizhskii et al; 2003). Proteins that contained similar peptides and could not be 623 differentiated based on MS/MS analysis alone were grouped to satisfy the principles 624 of parsimony. Proteins sharing significant peptide evidence were grouped into clusters. The final list of 542 proteins is provided in Table S1.

\section{Protein classification}

627 Flybase IDs of the entire list (542 proteins) were analysed in FlyMine. The tool for 628 pathway enrichment was used, with normalization to gene length, and Benjamini629 Hochberg correction factor of maximum $p$ value 0.05 . Most proteins were not 630 assigned to any pathways. In such cases, information from Uniprot and FlyBase were 631 used to assign a broad category for the protein. Most metabolic proteins were 632 assigned by FlyMine. Many proteins were involved in more than one pathway, as 633 apparent in the Table S1. Certain proteins, for example Gld and Glob1, although 634 known to be involved in redox homeostasis, were not annotated by FlyMine. In such 
635 cases they were assigned to a class manually. The human orthologues are from

636 FlyBase/DIOPT.

\section{Bioinformatic analysis (proteomics)}

638 The peptide counts for each protein across the diets tested were compiled into a 639 excel file with individual proteins represented in rows, and each sample per diet in 640 columns. Normalisation to mean of sum of all protein counts was performed.

641 Statistical analyses were performed in R Ver. 3.6.3. Significant changes were 642 analysed with a linear model fit in limma ver 3.42.2 (Ritchie et al., 2015) and edgeR 643 ver. 3.28.1 (McCarthy, Chen, \& Smyth, 2012) and adjusted with Benjamini Hochberg 644 for adjusted $p$ values. $30 \mathrm{~g} / \mathrm{L}$ was used as intercept in design. Heatmaps were 645 generated using heatmapper (Babicki et al., 2016) hierarchical clustering based on 646 Pearson complete distance. All other statistics were done using GraphPad prism 647 (8.3.0).

\section{GO term analyses}

649 GO terms for biological processes were assigned using PantherDB (Mi et al., 2021) 650 with the statistical enrichment tool. Gene names from each cluster (Figure 1D) were 651 separately analysed, and the list was sorted based on fold enrichment with reference 652 to the entire genome of Drosophila. The fold enrichment was converted to $\log _{2} \operatorname{values}$ 653 and plotted with GO terms as a bar graph using GraphPad prism (8.3.0).

\section{Measurement of ROS by microscopy}

655 After paternal diet intervention for 2 days, seminal vesicles were dissected (from $\mathrm{Dj}$ -

656 GFP or $\mathrm{W}^{1118}$ ) in insect medium (Sigma \# T3160) (for CellROX®) or 1X PBS

$657\left(\mathrm{H}_{2} \mathrm{DCFDA}\right)$, and subsequently incubated in CellROX® orange dye (Invitrogen \# $658 \mathrm{C} 10443)$ for 30 minutes at $37^{\circ} \mathrm{C}(5 \mu \mathrm{M}$ final concentration in insect medium) or $659 \mathrm{H}_{2}$ DCFDA (Invitrogen \#D399) at room temperature for 5 minutes (40 $\mu \mathrm{M}$ final 660 concentration). Following this, the tissue was rinsed extensively with $1 \mathrm{X}$ PBS and 661 mounted onto glass slides with halocarbon oil (HC700, Sigma) as the mounting 662 medium, and covered with glass coverslips. Coverslips were sealed with clear nail 663 polish and slides were imaged using an inverted confocal microscope (LSM800, 664 Zeiss) using absorption/emission maxima of $\sim 545 / 565 \mathrm{~nm}$ (for CellROX®) or 
665

666

667

668

669

670

671

672

673

674

675

676

677

678

679

680

681

682

683

684

685

686

687

688

689

690

691

692

693

$\sim 488 / 517 \mathrm{~nm}$ (for $\mathrm{H}_{2}$ DCFDA and GFP). All images were quantified in Fiji (Schindelin et al., 2012). The steps followed to process the images in Fiji are depicted below.

$>>F i j i$

>>image import .czi

>>process>subtract background>rolling ball radius 100 pixels

>>Image>adjust>threshold-set threshold min and max: 30-35 to 255

>>choose ROI

>>measure: Integrated density and mean gray values

\section{Antioxidant supplementation to counteract ROS}

$N$ acetyl cysteine (Sigma \# A7250) solubilized in water was used in food containing 30- or $300-\mathrm{g} / \mathrm{L}$ sugar at a final concentration of $1 \mathrm{mg} / \mathrm{mL}$, and intervention was carried out for two days at $26^{\circ} \mathrm{C}$. Following this, testes were dissected, and seminal vesicles were imaged for ROS production using CellROX® orange dye as described above. Simultaneously, sperm from 15 flies from each condition was isolated and used for sRNA sequencing as described below.

\section{RNAi crosses}

RNAi was initiated in the male germline by crossing males expressing UAS-RNAi (SOD1: BDSC\#29389; elF4A: BDSC\#33970; CCT4: BDSC\#77358) or UAS-GFP RNAi (BDSC\#35786) with virgin Nanos Gal4; UAS Dicer 2 (BDSC\#25751) on standard food. Eclosed F1 males were subjected to dietary intervention and ROS measurements using CellROX® orange dye as described above.

\section{Imaging for redox analysis}

Flies expressing roGFP2-orp1 in the mitochondria (BDSC\# 67672) were subjected to dietary intervention as detailed previously. Flies were dissected in 1X PBS and testes were mounted on halocarbon oil and sealed with coverslips. Imaging was performed immediately using confocal microscopy (upright LSM 700, Zeiss). The probe fluorescence was excited at 405nm and 488nm, sequentially and line by line with emission wavelengths ranging between 518-580 $\mathrm{nm}$. To determine the dynamic range, testes from the $30 \mathrm{~g} / \mathrm{L}$ sugar diet condition were either fully oxidised (using 
$69410 \% \mathrm{H}_{2} \mathrm{O}_{2}$ ) or fully reduced (1mM DTT) and immediately imaged with the above

695 settings.

\section{Quantification of redox changes}

697 Fiji was used to quantify each channel (405 nm and $488 \mathrm{~nm}$ ) separately, with

698 background subtraction and thresholding as described before. To calculate the

699 fluorescent intensity ratios, the $405 \mathrm{~nm}$ value was divided by $488 \mathrm{~nm}$. All ratios were

700 computed in Excel. The dynamic range (DR), which reflects the maximal achievable

701 redox changes in our model, was calculated by dividing the 405/488 ratio of the fully

702 oxidized $\mathrm{H}_{2} \mathrm{O}_{2}$ sample with the same ratio of the fully reduced DTT sample.

\section{Staining of testes with MitoTracker ${ }^{\mathrm{TM}}$ Red CMXRos}

704 Testes of 1- to 3-day-old males were dissected in PBS and stained in MitoTracker ${ }^{\mathrm{TM}}$

705 Red CMXRos in PBS (Molecular Probes, Eugene, OR, USA) for 15 minutes at room

706 temperature (final concentration $1 \mu \mathrm{M}$ ). Tissues were rinsed in 1XPBS and fixed in

$7074 \%$ paraformaldehyde solution for 10 minutes at room temperatures. Following

708 fixation, the tissues were extensively rinsed in 1X PBS triton (0.2\%) and mounted on

709 glass slides with VECTASHIELD® containing DAPI (Vectorlabs, H-1200-10).

710 Coverslips were sealed and imaging was performed in an inverted LSM 800 confocal

711 microscope with Texas red filter settings (ex/em: 579/599 nm). Quantification was

712 done after background subtraction and thresholding in Fiji as described before.

\section{Isolation of sperm for small RNA sequencing}

714 Sperm was isolated in TC-100 Insect Medium (Sigma\# T3160) essentially as

715 described in (Öst et al., 2014). From each diet, sperm from 15 flies were dissected

716 and pooled in 1:10 dilution of RNAse inhibitor (Recombinant ribonuclease inhibitor 5

717000 U, Cat. 2313A Takara), and samples were flash-frozen on dry-ice, and later

718 stored at $-80^{\circ} \mathrm{C}$. For sperm collection, five samples of each diet were prepared.

\section{Small RNA Library preparation and sequencing}

720 RNA extraction was done using miRNeasy Micro kit (Qiagen, 217084) according to

721 manufactors instructions. Prior to homogenization cold steel beads (0,15 g, SSB02-

722 RNA NextAdvance, Troy NY) were added to frozen samples followed by the addition 
of 500 ul of prechilled Qiazol (Qiagen). Samples were run in Tissue Lyser LT (Qigen) for $2+2$ min at 40 oscillations/second.

725

726

727

728

729

730

731

732

733

734

735

736

737

738

739

740

741

742

743

744

745

746

747

748

749

750

751

752

753

RNA quailty was studied with BioAnalyzer RNA analysis (5067-1511, Agilent, RNA 6000 nano kit). Small RNA libraries were produced with NEBNext Multiplex

SmallRNA Library Prep Kit for Illumina (E7560S, E7580, New England Biolabs) with the customisation of a dilution of primers 1:2. The 3'adaptor ligation reaction was carried out at $4^{\circ} \mathrm{C}$ overnight. To minimize the amount of $2 \mathrm{~S}$ rRNA we added a blocking oligo (5'-TAC AAC CCT CAA CCA TAT GTA GTC CAA GCA-SpcC3 3') to the samples at the $5^{\prime}$ adaptor ligation step ((Wickersheim \& Blumenstiel, 2013). Libraries were amplified for 16 cycles and cleaned using Agencourt AMPure XP (Beckman Coulter, Brea, CA). Size selection on amplified libraries was done using TBE gel (EC6265BOX, Invitrogen) 130-165 nt length. Extraction of cDNA from gel was performed with Gel Breaker Tubes (3388-100, IST Engineering) by incubation with buffer included in the NEBNext kit and incubated on a shaker for 1 hour at 37 degrees Celsius, flash frozen for 15 minutes and again incubated on shaker. Gel debris was removed by Spin-X 0,45 $\mu \mathrm{m}$ centrifuge tubes (Corning Inc., Corning, NY). Percipitation was done using GlycoBlue (Invitrogen), 0,1 times the volume of Acetate $3 \mathrm{M}(\mathrm{pH} 5.5)$, and 3 times the volume of $100 \%$ Ethanol in $-70^{\circ} \mathrm{C}$ overnight. Quality of cDNA libraries were studied with BioAnalyzer DNA analysis (5067-1504, Agilent, Agilent High Sensitivity DNA kit, 5067-4626). Final DNA concentration was determined with Quantus Fluorometer (E6150, Promega, Madison, WI) using QuantiFluor ONE ds DNA system. Libraries were pooled and sequenced on NextSeq 500 with NextSeq 500/550 High Output kit version 2.5, 75 cycles (Illumina, San

Diego, CA). All libraries passed Illumina's default quality control.

\section{Bioinformatic analyses (sRNA)}

Data analysis was performed with Seqpac ver. 0.99.0 (Skog et al., 2021). Adaptor trimming, quality control and mapping were all performed in Seqpac with make_counts and make_reanno workflow with an evidence of that an individual sequence should have at least 1 count in 2 separate samples. Trimming was performed on the adaptor sequence of the used NebNext library (AGATCGGAAGAGCACACGTCTGAACTCCAGTCA). Only reads with an adaptor 
sequence present prior to trimming were studied. Averaged over all 20 samples, $1.9^{*} 10^{6}$ unique reads passed filtering and with a mean of $1,5^{*} 10^{7}$ reads per sample.

Genomic mapping was performed towards Drosophila reference dm6 downloaded from UCSC. Biotype mapping was performed to Ensembl ncRNA BDGP6.32, piRNA piRBase D. Melanogaster 2.0 (J. Wang et al., 2019; Yuan et al., 2016; P. Zhang et al., 2014, 2015), miRBase 21 (Griffiths-Jones, 2004; Griffiths-Jones, Grocock, van Dongen, Bateman, \& Enright, 2006; Griffiths-Jones, Saini, Van Dongen, \& Enright, 2008; Kozomara, Birgaoanu, \& Griffiths-Jones, 2019; Kozomara \& Griffiths-Jones, 2011, 2014), protein coding from Ensembl BDGP6.32 (Howe et al., 2021)and tRNA from GtRNAdb (Chan \& Lowe, 2009, 2016) Drosophila BDGP dm6. Human mapping was performed against the human genome GRCh38.p13 (GCA_000001405.28) from Ensembl. Biotype mapping was performed to Ensembl ncRNA, piRNA piRBase v 2.0, miRBase 21, protein coding from NCBI RefSeq proteins and tRNA from GtRNAdb. Biotypes were hierarchically determined in the order rRNA, mitochondrial tRNA, tRNA, miRNA, snoRNA, IncRNA, snRNA, piRNA and protein coding. Drosophila mitochondrial genome NC_024511.2 was downloaded from NCBI. Bowtie indexes were created with Rbowtie ver 1.32.0.

Data was first filtered with function PAC_filter on a size of 18-50 nt length, 10 counts in $60 \%$ of samples and a perfect (no mismatch) match with reference genome. Additional filtering was performed after CPM calculations to remove reads without a presence of minimum 20 counts per million in $25 \%$ of samples. Computation of counts per million, log fold change and Figure 3 B, C, E, F and Figure 4 B and D were generated with Seqpac. Other figures presented were created with ggplot2 ver 3.3.3 and pheatmap ver 1.0.12. Unless stated otherwise, data used in figures are CPM for individual sequences. In figure $3 \mathrm{E}, \log _{2}$ fold change is calculated on a feature-base rather than sequence base, where all sequences mapping to a certain miRNA are classified together.

Analysis of tsRNA and their cleavage sites were performed with the PAC_mapper and PAC_trna analytic workflow in Seqpac. Here, ss-files constructed with tRNAscan-SE for the Drosophila nuclear and mitochondrial tRNA were used (Lowe \& Chan, 2016). We defined five tsRNA subtypes; 5'-half, 5'-tsRNA, i-tsRNA, 3'-tsRNA and 3'-half, where a 5'-half starts in the 5' end of the mature tRNA and ends in the 
antiocodon loop. Furthermore, 5'-tsRNA also starts in the 5' end but ends prior to the antiocodon loop. The opposite relationship is true for 3'-halves and 3'-tsRNA, whilst itsRNA are fragments without connection to either 5' nor 3' end. Fragments from tRNA are here reported to the isodecoder and isoacceptor they originate from, as most of nuclear tsRNA maps to several copies at once. This multimapping is found in Table S4.

For all source code used, see https://github.com/signeskog/Ramesh-2021.

\section{Statistical analyses (sRNA)}

Statistics on sRNA data were performed with a Negative Binomial Generalized Linear Model, since the data are counts based. sRNA sequences are in some cases impossible to map to one unique place in the genome, due to their short size and the repetitive nature of some transcription sites. As of now, there is no perfect method to add this uncertainty into a statistical model and we have not accounted for the ambiguity stemming from the risk of multimapping. Since we cannot guarantee that each sequence stems from one original place, we did not perform multiple testing.

In the case of miRNAs, we did not take miRNA isoforms into account, but rather combined sequences originating from the same miRNA. This made it so our model studied the difference on a miRNA-to-miRNA basis rather than sequence to sequence basis. The model was performed with function glm.nb from the $\mathrm{R}$ package MASS version 7.3-51.4. Clustering of tsRNA (Figure $4 \mathrm{C}$ ) were performed with pheatmap $v$ 1.0.12, where clustering was performed on rows with $\mathrm{k}=4$ and with Euclidean distance. Standard error of the mean for $\log _{2}$ fold changes were calculated on the SEM of each individual of $30 \mathrm{~g} / \mathrm{L}(\mathrm{n}=5)$ fold change against $300 \mathrm{~g} / \mathrm{L}$ for each sequence.

\section{Resource availability}

\section{Lead contact}

Further questions may be sent to lead contact Anita Öst (anita.ost@liu.se).

\section{Materials availability}

14 All Drosophila strains used in this manuscript are commercially available. 
816 Data and code availability

817 Raw sRNA-seq performed in sperm have been deposited at Sequence Read Archive

818 (SRA) at accession number PRJNA770968. All code for this project is available on

819 GitHub at https://github.com/signeskog/Ramesh-2021.

820 Supplementary information

\section{Figure S1: Proteomic composition of Drosophila sperm}

822 A: The nine most abundant proteins are sperm specific and make up a third of the

823 total counts. These sperm specific abundant proteins are indicated in orange within

824 the pie-chart. Total counts distribution is presented.

825 B: The expression of the top 9 abundant sperm proteins from $\mathbf{A}$ is not affected by

826 dietary sugar as shown in the bar graph. Mean $\log _{2}$ values are plotted in the bar

827 graph.

828 C: Schematics of RNAi crossing scheme. RNAi was induced in the germline by

829 crossing virgin female Nanos-Gal4; UAS-Dicer2 flies with males carrying the UAS-

830 RNAi construct. The F1 adults were screened for testes phenotype, and subjected to

831 ROS measurements in seminal vesicles using ROS indicators

832 D: Light-microscopy image of testes phenotype with and without elF4A in the

833 germline. DIC images of Nanos Gal4; Dicer2 heterozygous testes shows normal

834 testes (T), accessory glands (AG) and seminal vesicles with sperm (SV). Nanos-

835 Gal4; Dicer2> elF4A RNAi (elF4Ai) shows shorter T and lack SV. Scale bar: $700 \mu \mathrm{m}$

$836 \mathrm{DIC}=$ differential interference contrast

837 Related to Figure 1

838 Figure S2:

839 A: The mito-roGFP2-Orp1 is expressed and visualised in seminal vesicles (top panel) 840 and on sperm (bottom panel). The sequential excitation wavelengths are 405- and $841488 \mathrm{~nm}$ as indicated, and emission was captured at 518-580 nm. Scale bar: $50 \mu \mathrm{m}$

842 B: Establishment of dynamic range for measurement of redox changes using the 843 mito-roGFP2-Orp1 sensor. $\mathrm{H}_{2} \mathrm{O}_{2}$ and DTT represent fully oxidised and fully reduced 844 conditions, respectively. Bar graphs represent quantified ratio of fluorescence 845 emission from sequential excitation at 405-and $488 \mathrm{~nm}$. Reduced condition was 846 arbitrarily set at 0.2 . $\mathrm{DR}=$ dynamic range. Scale bar: $50 \mu \mathrm{m}$

847 C: Live imaging of roGFP2-Orp1 in sperm/seminal vesicles after dietary intervention 
849 D: MitoTracker ${ }^{\mathrm{TM}}$ Red-CMRos staining reveals no apparent change in mitochondrial 850 potential $(\Delta \psi \mathrm{m})$ in sperm/ seminal vesicle. Flies were fed 30 - or $300 \mathrm{~g} / \mathrm{L}$ sugar diet

851 for two days, seminal vesciles were dissected, stained with the dye, and subjected to 852 paraformaldehyde fixation. Fluorescence microscopy images showing cellular

853 distribution of mitochondria-specific dye MitoTracker ${ }^{\mathrm{TM}}$ Red (red) in the sperm within 854 the seminal vesicle. The tissues were counter stained for nuclei using Hoescht stain 855 (White) and representative images for both diets are presented. Scale bar: $50 \mu \mathrm{m}$

856 E: Quantification of ROS labelled with $\mathrm{H}_{2}$ DCFDA: The same flies in D were

857 dissected, and seminal vesicles were immediately incubated with the ROS labelling

858 dye- $\mathrm{H}_{2}$ DCFDA and imaged. The micrographs were quantified and fluorescence (a.u)

859 are plotted. The data shown are mean \pm SEM. $n=20$, asterisks $\left({ }^{*}\right)$ represent $p \leq$ 860 0.001, unpaired t-test.

861 Related to Figure 2

862 Figure S3: Biotype distribution in sperm is changed by dietary intervention

863 A: Fold changes $30-$ vs $300 \mathrm{~g} / \mathrm{L}$ of the indicated sRNA biotypes are presented. Each 864 dot represents a unique transcript. The reads mapping to nuclear and mitochondrial 865 genome are separated.

866 B: Fold changes $30 \mathrm{~g} / \mathrm{L}$ vs $30 \mathrm{~g} / \mathrm{L}$ with NAC of the indicated sRNA biotypes are 867 presented. Each dot represents a unique transcript. The reads mapping to nuclear 868 and mitochondrial genome are separated.

869 C: Dietary change of miRNA in $30 \mathrm{~g} / \mathrm{L}$ sugar vs $300 \mathrm{~g} / \mathrm{L}$. All miRNA identified in the 870 study in fly sperm are presented under the fly pictogram. The corresponding human 871 orthologues, where present, are indicated under the human pictogram. Bar graphs in 872 descending order represent abundance ( $\left.\log _{10} \mathrm{CPM}\right)$ of each miRNA. Each miRNA 873 represents a mean of all transcripts annotated to that miRNA. Fold changes between $87430-$ vs $300 \mathrm{~g} / \mathrm{L}$ are indicated as closed circles. Asterisk $\left({ }^{*}\right)$ represents statistically 875 significant miRNA $(p<0.05)$ calculated by Generalized Linear Negative Binomial 876 model, error bars are standard error (SE).

877 Related to Figure 3

878 Figure S4: Distribution of tsRNA cleavage sites in cellular compartments

879 A: Mean CPM of all tsRNA, categorised on cleavage site. Each stacked bar 880 represents one dietary condition. 
B: Mean CPM of tsRNA, categorised by cleavage site. Clusters 1-4 represent the

882

883

884

885

886

887

888

889

890

891

892

893

894

895

896

897

898

899

900

901

902

903

904

905

906

907

908

909

910

911

912

913

914

915

916

917

918 hierarchical clustering as described for Figure 4C.
C: Percentage of mean CPM of tsRNA, categorised by cleavage site. Stacked bars

are divided by what hierarchical cluster they were classified as, according to heatmap in Figure 4C.

Related to Figure 4

\section{References}

Abu-Halima, M., Backes, C., Leidinger, P., Keller, A., Lubbad, A. M., Hammadeh, M., \& Meese, E. (2014). MicroRNA expression profiles in human testicular tissues of infertile men with different histopathologic patterns. Fertility and Sterility, 101(1). https://doi.org/10.1016/j.fertnstert.2013.09.009

Agarwal, A., Makker, K., \& Sharma, R. (2008). Clinical relevance of oxidative stress in male factor infertility: An update. American Journal of Reproductive Immunology, Vol. 59, pp. 2-11. https://doi.org/10.1111/j.1600-0897.2007.00559.x

Agarwal, A., Parekh, N., Selvam, M. K. P., Henkel, R., Shah, R., Homa, S. T., Ramaswamy, R., Ko, E., Tremellen, E., Estervaes, S., Majroub, A., et al (2019). Male oxidative stress infertility (MOSI): Proposed terminology and clinical practice guidelines for management of idiopathic male infertility. World Journal of Mens Health, Vol. 37, pp. 296-312. https://doi.org/10.5534/wjmh.190055

Agarwal, A., Selvam, M. K. P., \& Baskaran, S. (2020). Proteomic analyses of human sperm cells: Understanding the role of proteins and molecular pathways affecting male reproductive health. International Journal of Molecular Sciences, Vol. 21. https://doi.org/10.3390/ijms21051621

Agarwal, A., Sharma, R., Samanta, L., Durairajanayagam, D., \& Sabanegh, E. (2016). Proteomic signatures of infertile men with clinical varicocele and their validation studies reveal mitochondrial dysfunction leading to infertility. Asian Journal of Andrology, 18(2), 282-291. https://doi.org/10.4103/1008-682X.170445

Albrecht, S. C., Barata, A. G., Großhans, J., Teleman, A. A., \& Dick, T. P. (2011). In vivo mapping of hydrogen peroxide and oxidized glutathione reveals chemical and regional specificity of redox homeostasis. Cell Metabolism, 14(6), 819-829.

https://doi.org/10.1016/j.cmet.2011.10.010

Amaral, A., Paiva, C., Attardo Parrinello, C., Estanyol, J. M., Ballescà, J. L., Ramalho-Santos, J., \& Oliva, R. (2014). Identification of proteins involved in human sperm motility using high-throughput differential proteomics. Journal of Proteome Research, 13(12), 5670-5684. https://doi.org/10.1021/pr500652y

Babicki, S., Arndt, D., Marcu, A., Liang, Y., Grant, J. R., Maciejewski, A., \& Wishart, D. S. (2016). Heatmapper: web-enabled heat mapping for all. Nucleic Acids Research, 44(W1), W147-W153. https://doi.org/10.1093/nar/gkw419 
919 Barati, E., Nikzad, H., \& Karimian, M. (2020). Oxidative stress and male infertility: current knowledge of pathophysiology and role of antioxidant therapy in disease management. Cellular and Molecular Life Sciences, Vol. 77, pp. 93-113. https://doi.org/10.1007/s00018-019-03253-8

923

924

925

926

927

928

929

930

931

932

933

934

935

936

937

938

939

940

941

942

943

944

945

946

947

948

949

950

951

952

953

954

955

956

957

958

959

Barbu, M. G., Thompson, D. C., Suciu, N., Voinea, S. C., Cretoiu, D., \& Predescu, D. V. (2021). The roles of micrornas in male infertility. International Journal of Molecular Sciences, Vol. 22, pp. 1-13. https://doi.org/10.3390/ijms22062910

Barceló, M., Mata, A., Bassas, L., \& Larriba, S. (2018). Exosomal microRNAs in seminal plasma are markers of the origin of azoospermia and can predict the presence of sperm in testicular tissue. Human Reproduction, 33(6), 1087-1098. https://doi.org/10.1093/humrep/dey072

Bastian, F. B., Roux, J., Niknejad, A., Comte, A., Fonseca Costa, S. S., de Farias, T. M., Moretti, S., Parmetier, G., de Laval, V. R., Rosikiewicz, M., et al (2021). The Bgee suite: integrated curated expression atlas and comparative transcriptomics in animals. Nucleic Acids Research, 49(D1), D831-D847.

https://doi.org/10.1093/NAR/GKAA793

Belleannée, C., Calvo, É., Caballero, J., \& Sullivan, R. (2013). Epididymosomes convey different repertoires of micrornas throughout the bovine epididymis. Biology of Reproduction, 89(2). https://doi.org/10.1095/biolreprod.113.110486

Boguenet, M., Bouet, P. E., Spiers, A., Reynier, P., \& May-Panloup, P. (2021). Mitochondria: Their role in spermatozoa and in male infertility. Human Reproduction Update, Vol. 27, pp. 697-719. https://doi.org/10.1093/humupd/dmab001

Bui, A. D., Sharma, R., Henkel, R., \& Agarwal, A. (2018). Reactive oxygen species impact on sperm DNA and its role in male infertility. Andrologia, Vol. 50. https://doi.org/10.1111/and.13012

Candenas, L., \& Chianese, R. (2020). Exosome composition and seminal plasma proteome: A promising source of biomarkers of male infertility. International Journal of Molecular Sciences, Vol. 21, pp. 1-27. https://doi.org/10.3390/ijms21197022

Carrell, D. T., Aston, K. I., Oliva, R., Emery, B. R., \& De Jonge, C. J. (2016). The "omics" of human male infertility: integrating big data in a systems biology approach. Cell and Tissue Research, Vol. 363, pp. 295-312. https://doi.org/10.1007/s00441015-2320-7

Cescon, M., Chianese, R., \& Tavares, R. S. (2020). Environmental impact on male (in)fertility via epigenetic route. Journal of Clinical Medicine, Vol. 9, pp. 1-33. https://doi.org/10.3390/jcm9082520

Chan, P. P., \& Lowe, T. M. (2009). GtRNAdb: A database of transfer RNA genes detected in genomic sequence. Nucleic Acids Research, 37(SUPPL. 1), D93-D97. https://doi.org/10.1093/nar/gkn787

Chan, P. P., \& Lowe, T. M. (2016). GtRNAdb 2.0: An expanded database of transfer RNA genes identified in complete and draft genomes. Nucleic Acids Research, 44(D1), D184-D189. https://doi.org/10.1093/nar/gkv1309 
960 Chen, Q., Yan, M., Cao, Z., Li, X., Zhang, Y., Shi, J., feng, G. H., Peng, H., Zhang, 961 X., Zhang, Y., et al (2016). Sperm tsRNAs contribute to intergenerational inheritance 962 of an acquired metabolic disorder. Science, 351(6271), 397-400.

963 https://doi.org/10.1126/science.aad7977

964 Chianese, R., \& Pierantoni, R. (2021). Mitochondrial reactive oxygen species (ROS)

965

966

967

968

969

970

971

972

973

974

975

976

977

978

979

980

981

982

983

984

985

986

987

988

989

990

991

992

993

994

995

996

997

998

999

1000 production alters sperm quality. Antioxidants, Vol. 10, pp. 1-19. https://doi.org/10.3390/antiox10010092

Chianese, R., Troisi, J., Richards, S., Scafuro, M., Fasano, S., Guida, M., Pierantoni, R., Meccariello, R. (2017). Bisphenol A in Reproduction: Epigenetic Effects. Current Medicinal Chemistry, 25(6), 748-770.

https://doi.org/10.2174/0929867324666171009121001

Cropley, J. E., Eaton, S. A., Aiken, A., Young, P. E., Giannoulatou, E., Ho, J., Buckland, M. E., Keam, S. P., Hutvagner, G., Humphreys, D. T., Langley, K. G., Henstridge, D. C., Martin, D., Febbraio, M. A., \& Suter, C. M. (2016). Male-lineage transmission of an acquired metabolic phenotype induced by grand-paternal obesity. Molecular metabolism, 5(8), 699-708. https://doi.org/10.1016/j.molmet.2016.06.008

D’Elia, D., Catalano, D., Licciulli, F., Turi, A., Tripoli, G., Porcelli, D., ... Caggese, C. (2006). The MitoDrome database annotates and compares the OXPHOS nuclear genes of Drosophila melanogaster, Drosophila pseudoobscura and Anopheles gambiae. Mitochondrion, 6(5), 252-257. https://doi.org/10.1016/J.MITO.2006.07.001

Day, J., Savani, S., Krempley, B. D., Nguyen, M., \& Kitlinska, J. B. (2016). Influence of paternal preconception exposures on their offspring: Through epigenetics to phenotype. American Journal of Stem Cells, Vol. 5, pp. 11-18. Retrieved from https://pubmed.ncbi.nlm.nih.gov/27335698/

Donkin, I., Versteyhe, S., Ingerslev, L. R., Qian, K., Mechta, M., Nordkap, L., Mortensen, B., Appel, E. V., Jørgensen, N., Kristiansen, V. B. et al (2016). Obesity and bariatric surgery drive epigenetic variation of spermatozoa in humans. Cell Metabolism, 23(2), 369-378. https://doi.org/10.1016/j.cmet.2015.11.004

Dorus, S., Busby, S. A., Gerike, U., Shabanowitz, J., Hunt, D. F., \& Karr, T. L. (2006). Genomic and functional evolution of the Drosophila melanogaster sperm proteome. Nature Genetics, 38(12), 1440-1445. https://doi.org/10.1038/ng1915

Du Plessis, S. S., Agarwal, A., Halabi, J., \& Tvrda, E. (2015). Contemporary evidence on the physiological role of reactive oxygen species in human sperm function. Journal of Assisted Reproduction and Genetics, 32(4), 509-520. https://doi.org/10.1007/s10815-014-0425-7

Du Plessis, S. S., Cabler, S., McAlister, D. A., Sabanegh, E., \& Agarwal, A. (2010). The effect of obesity on sperm disorders and male infertility. Nature Reviews Urology, Vol. 7, pp. 153-161. https://doi.org/10.1038/nrurol.2010.6

Dutta, S., Henkel, R., Sengupta, P., \& Agarwal, A. (2020). Physiological Role of ROS in Sperm Function. In Male Infertility (pp. 337-345). https://doi.org/10.1007/978-3030-32300-4_27 
1001 Evans, E., Scholten, J., Mzyk, A., Reyes-San-Martin, C., Llumbet, A. E., Hamoh, T.,

1002 Arts, E., Schirhagl, R., \& Cantineau, A. (2021). Male subfertility and oxidative stress.

1003 Redox Biology, Vol. 46. https://doi.org/10.1016/j.redox.2021.102071

1004 Fullston, T., Ohlsson-Teague, E. M. C., Print, C. G., Sandeman, L. Y., \& Lane, M. 1005 (2016). Sperm microRNA content is altered in a mouse model of male obesity, but 1006 the same suite of microRNAs are not altered in offspring's sperm. PLoS ONE, 11(11). https://doi.org/10.1371/journal.pone.0166076

1008 Fullston, T., Ohlsson Teague, E. M., Palmer, N. O., DeBlasio, M. J., Mitchell, M., 1009 Corbett, M., Print, C. G., Owens, J. A., \& Lane, M. (2013). Paternal obesity initiates 1010 metabolic disturbances in two generations of mice with incomplete penetrance to the 1011 F2 generation and alters the transcriptional profile of testis and sperm microRNA 1012 content. FASEB Journal, 27(10), 4226-4243. https://doi.org/10.1096/fj.12-224048

1013 Gao, H., Wen, H., Cao, C., Dong, D., Yang, C., Xie, S., Zhang, J., Huang, X., Huang, X., Yuan, S., \& Dong, W. (2019). Overexpression of microrna-10a in germ cells causes male infertility by targeting rad51 in mouse and human. Frontiers in Physiology, 10. https://doi.org/10.3389/fphys.2019.00765

1017 Gapp, K., \& Miska, E. A. (2016). TRNA fragments: Novel players in intergenerational 1018 inheritance. Cell Research, Vol. 26, pp. 395-396. https://doi.org/10.1038/cr.2016.24

1019 Gapp, K., Parada, G. E., Gross, F., Corcoba, A., Kaur, J., Grau, E., hemberg, M., 1020 Bohacek, J., \& Miska, E. A. (2021). Single paternal dexamethasone challenge programs offspring metabolism and reveals multiple candidates in RNA-mediated inheritance. IScience, 24(8). https://doi.org/10.1016/j.isci.2021.102870

1023 Gapp, K., Soldado-Magraner, S., Alvarez-Sánchez, M., Bohacek, J., Vernaz, G., Shu, H.,Franklin, T.B., Wolfer, D., \& Mansuy, I. M. (2014). Early life stress in fathers improves behavioural flexibility in their offspring. Nature Communications, 5(1), 1-8. https://doi.org/10.1038/ncomms6466

1027 Grandjean, V., Fourré, S., De Abreu, D. A. F., Derieppe, M. A., Remy, J. J., \& 1028 Rassoulzadegan, M. (2015). RNA-mediated paternal heredity of diet-induced obesity and metabolic disorders. Scientific Reports, 5. https://doi.org/10.1038/srep18193

1030 Griffiths-Jones, S. (2004). The microRNA registry. Nucleic Acids Research, 32(DATABASE ISS.), D109-D111. https://doi.org/10.1093/nar/gkh023

1032 Griffiths-Jones, S., Grocock, R. J., van Dongen, S., Bateman, A., \& Enright, A. J. 1033 (2006). miRBase: microRNA sequences, targets and gene nomenclature. Nucleic 1034 Acids Research, 34(Database issue), D140-D144. https://doi.org/10.1093/nar/gkj112

1035 Griffiths-Jones, S., Saini, H. K., Van Dongen, S., \& Enright, A. J. (2008). miRBase: 1036 Tools for microRNA genomics. Nucleic Acids Research, 36(SUPPL. 1), D154-D158. 1037 https://doi.org/10.1093/nar/gkm952

1038 Grosso, J. B., Zoff, L., Calvo, K. L., Maraval, M. B., Perez, M., Carbonaro, M., 1039 Brignardello, C., Morente, C., \& Spinelli, S. V. (2021). Levels of seminal tRNA1040 derived fragments from normozoospermic men correlate with the success rate of 
1041 ART. Molecular Human Reproduction, 27(4), 1-12.

1042 https://doi.org/10.1093/molehr/gaab017

1043 Holmström, K. M., \& Finkel, T. (2014). Cellular mechanisms and physiological consequences of redox-dependent signalling. Nature Reviews Molecular Cell Biology, Vol. 15, pp. 411-421. https://doi.org/10.1038/nrm3801

Howe, K. L., Achuthan, P., Allen, J., Allen, J., Alvarez-Jarreta, J., Amode, M. R., Armean, I. M., Azov, A. G., Bennett, R., Bhai, J.,et al. (2021). Ensembl 2021. Nucleic Acids Research, 49(D1), D884-D891. https://doi.org/10.1093/nar/gkaa942 (2019). Identification of small non-coding RNAs as sperm quality biomarkers for in vitro fertilization. Cell Discovery, Vol. 5, pp. 1-5. https://doi.org/10.1038/s41421-0190087-9

1054

1055

1056

1057

1058

1059

1060

1061

1062

1063

1064

1065

1066

1067

1068

1069

1070

1071

1072

1073

1074

1075

1076

1077

1078

1079

Hudry, B., de Goeij, E., Mineo, A., Gaspar, P., Hadjieconomou, D., Studd, C., Mokochinski, J. B., Kramer, H. B., Plaçais, P. Y., et al (2019). Sex Differences in Intestinal Carbohydrate Metabolism Promote Food Intake and Sperm Maturation. Cell, 178(4), 901-918.e16. https://doi.org/10.1016/j.cell.2019.07.029

Jodar, M., Soler-Ventura, A., \& Oliva, R. (2017). Semen proteomics and male infertility. Journal of Proteomics, Vol. 162, pp. 125-134.

https://doi.org/10.1016/j.jprot.2016.08.018

Katib, A. (2015). Mechanisms linking obesity to male infertility. Central European Journal of Urology, 68(1), 79-85. https://doi.org/10.5173/ceju.2015.01.435

Kiani, M., Salehi, M., \& Mogheiseh, A. (2019). MicroRNA expression in infertile men: Its alterations and effects. Zygote, Vol. 27, pp. 263-271.

https://doi.org/10.1017/S0967199419000340

Koppers, A. J., De luliis, G. N., Finnie, J. M., McLaughlin, E. A., \& Aitken, R. J. (2008). Significance of Mitochondrial Reactive Oxygen Species in the Generation of Oxidative Stress in Spermatozoa. The Journal of Clinical Endocrinology \& Metabolism, 93(8), 3199-3207. https://doi.org/10.1210/JC.2007-2616

Kotaja, N. (2014, June 1). MicroRNAs and spermatogenesis. Fertility and Sterility, Vol. 101, pp. 1552-1562. https://doi.org/10.1016/j.fertnstert.2014.04.025

Kothari, S., Thompson, A., Agarwal, A., \& Plessis, S. S. du. (2010). Free radicals: their beneficial and detrimental effects on sperm function. Indian Journal of Experimental Biology, 48, 425-435.

Kozomara, A., Birgaoanu, M., \& Griffiths-Jones, S. (2019). MiRBase: From microRNA sequences to function. Nucleic Acids Research, 47(D1), D155-D162. https://doi.org/10.1093/nar/gky1141

Kozomara, A., \& Griffiths-Jones, S. (2011). MiRBase: Integrating microRNA annotation and deep-sequencing data. Nucleic Acids Research, 39(SUPPL. 1), D152-D157. https://doi.org/10.1093/nar/gkq1027 
1080 Kozomara, A., \& Griffiths-Jones, S. (2014). MiRBase: Annotating high confidence microRNAs using deep sequencing data. Nucleic Acids Research, 42(D1), D68-D73. https://doi.org/10.1093/nar/gkt1181

1083 Larriba, E., Rial, E., \& del Mazo, J. (2018). The landscape of mitochondrial small non-

1084

1085

1086

1087

1088

1089

1090

1091

1092

1093

1094

1095

1096

1097

1098

1099

1100

1101

1102

1103

1104

1105

1106

1107

1108

1109

1110

1111

1112

1113

1114 coding RNAs in the PGCs of male mice, spermatogonia, gametes and in zygotes. BMC Genomics, 19(1), 1-12. https://doi.org/10.1186/s12864-018-5020-3

Lempradl, A., Kugelberg, U., Iconomou, M., Beddows, I., Nätt, D., Casas, E., Örkenby, L., Enders, L., Martinez, A.G., et al (2021). Intergenerational metabolic priming by sperm piRNAs. BioRxiv, 2021.03.29.436592.

https://doi.org/10.1101/2021.03.29.436592

Lian, J., Zhang, X., Tian, H., Liang, N., Wang, Y., Liang, C., Li, X., \& Sun, F. (2009). Altered microRNA expression in patients with non-obstructive azoospermia.

Reproductive Biology and Endocrinology, 7, 13. https://doi.org/10.1186/1477-7827-713

Liu, Y., \& Ding, Z. (2017). Obesity, a serious etiologic factor for male subfertility in modern society. Reproduction, Vol. 154, pp. R123-R131.

https://doi.org/10.1530/REP-17-0161

Lowe, T. M., \& Chan, P. P. (2016). tRNAscan-SE On-line: integrating search and context for analysis of transfer RNA genes. Nucleic Acids Research, 44(W1), W54W57. https://doi.org/10.1093/nar/gkw413

MacLeod, J. (1943). The role of oxygen in the metabolism and motility of human spermatozoa. American Journal of Physiology-Legacy Content, 138(3), 512-518. https://doi.org/10.1152/ajplegacy.1943.138.3.512

McCarthy, D. J., Chen, Y., \& Smyth, G. K. (2012). Differential expression analysis of multifactor RNA-Seq experiments with respect to biological variation. Nucleic Acids Research, 40(10), 4288-4297. https://doi.org/10.1093/nar/gks042

Mi, H., Ebert, D., Muruganujan, A., Mills, C., Albou, L. P., Mushayamaha, T., \& Thomas, P. D. (2021). PANTHER version 16: A revised family classification, treebased classification tool, enhancer regions and extensive API. Nucleic Acids Research, 49(D1), D394-D403. https://doi.org/10.1093/nar/gkaa1106

Molla-Herman, A., Angelova, M. T., Ginestet, M., Carré, C., Antoniewski, C., \& Huynh, J. R. (2020). tRNA Fragments Populations Analysis in Mutants Affecting tRNAs Processing and tRNA Methylation. Frontiers in Genetics, 11, 1158. https://doi.org/10.3389/fgene.2020.518949

Moscatelli, N., Lunetti, P., Braccia, C., Armirotti, A., Pisanello, F., De Vittorio, M., Zara, V., \& Ferramosca, A. (2019). Comparative proteomic analysis of proteins involved in bioenergetics pathways associated with human sperm motility. International Journal of Molecular Sciences, 20(12), 3000. https://doi.org/10.3390/ijms20123000

Muñoz, X., Mata, A., Bassas, L., \& Larriba, S. (2015). Altered miRNA Signature of Developing Germ-cells in Infertile Patients Relates to the Severity of Spermatogenic 
1121 Failure and Persists in Spermatozoa. Scientific Reports, 5.

1122 https://doi.org/10.1038/srep17991

1123 Nätt, D., \& Öst, A. (2020). Male reproductive health and intergenerational metabolic 1124 responses from a small RNA perspective. Journal of Internal Medicine, Vol. 288, pp. 305-320. https://doi.org/10.1111/joim.13096

1126 Nätt, D., Kugelberg, U., Casas, E., Nedstrand, E., Zalavary, S., Henriksson, P., Nijm, C., Jäderquist, J., Sandborg, J., Flinke, E., et al (2019). Human sperm displays rapid responses to diet. PLoS Biology, 17(12). https://doi.org/10.1371/journal.pbio.3000559

1129 Nesvizhskii, A. I., Keller, A., Kolker, E., \& Aebersold, R. (2003). A statistical model for 1130 identifying proteins by tandem mass spectrometry. Analytical Chemistry, 75(17),

1131 4646-4658. https://doi.org/10.1021/AC0341261

1132 Nixon, B., De luliis, G. N., Dun, M. D., Zhou, W., Trigg, N. A., \& Eamens, A. L. (2019, 1133 September 1). Profiling of epididymal small non-protein-coding RNAs. Andrology,

1134 Vol. 7, pp. 669-680. https://doi.org/10.1111/andr.12640

1135 Nixon, B., De luliis, G. N., Hart, H. M., Zhou, W., Mathe, A., Bernstein, I. R., 1136 Anderson, A. L., Stanger, S. J., Skerrett-Byrne, D. A., Jamaluddin, M., et al (2019).

1137 Proteomic profiling of mouse epididymosomes reveals their contributions to post1138 testicular sperm maturation. Molecular and Cellular Proteomics, 18, S91-S108. https://doi.org/10.1074/mcp.RA118.000946

1140 Nowicka-Bauer, K., Lepczynski, A., Ozgo, M., Kamieniczna, M., Fraczek, M., Stanski, L., Olszewska, M., Malcher, A., Skrzypczak, W., \& Kurpisz, M. K. (2018). Sperm mitochondrial dysfunction and oxidative stress as possible reasons for isolated asthenozoospermia. Journal of Physiology and Pharmacology, 69(3).

1144 https://doi.org/10.26402/jpp.2018.3.05 Öst, A., Lempradl, A., Casas, E., Weigert, M., Tiko, T., Deniz, M., Pantano, L., Boenisch, U., Itskov, P. M., Stoeckius, M., et al. (2014). Paternal Diet Defines Offspring Chromatin State and Intergenerational Obesity. Cell, 159(6), 1352-1364. https://doi.org/10.1016/J.CELL.2014.11.005

1149 Peng, H., Shi, J., Zhang, Y., Zhang, H., Liao, S., Li, W., Lei, L., Han, C., Ning, L., Cao, Y., et al (2012). A novel class of tRNA-derived small RNAs extremely enriched in mature mouse sperm. Cell Research, Vol. 22, pp. 1609-1612. https://doi.org/10.1038/cr.2012.141

1153 Pergialiotis, V., Prodromidou, A., Frountzas, M., Korou, L. M., Vlachos, G. D., \& Perrea, D. (2016, August 1). Diabetes mellitus and functional sperm characteristics: A meta-analysis of observational studies. Journal of Diabetes and Its Complications, Vol. 30, pp. 1167-1176. https://doi.org/10.1016/j.jdiacomp.2016.04.002

1157 Rhee, S. G. (2006, June 30). H2O2, a necessary evil for cell signaling. Science, Vol. 312, pp. 1882-1883. https://doi.org/10.1126/science.1130481

1159 Ritchie, M. E., Phipson, B., Wu, D., Hu, Y., Law, C. W., Shi, W., \& Smyth, G. K. 1160 (2015). Limma powers differential expression analyses for RNA-sequencing and 
1161

1162

1163

1164

1165

1166

1167

1168

1169

1170

1171

1172

1173

1174

1175

1176

1177

1178

1179

1180

1181

1182

1183

1184

1185

1186

1187

1188

1189

1190

1191

1192

1193

1194

1195

1196

1197

1198

1199

1200

microarray studies. Nucleic Acids Research, 43(7), e47.

https://doi.org/10.1093/nar/gkv007

Rodgers, A. B., Morgan, C. P., Leu, N. A., \& Bale, T. L. (2015). Transgenerational epigenetic programming via sperm microRNA recapitulates effects of paternal stress. Proceedings of the National Academy of Sciences of the United States of America, 112(44), 13699-13704. https://doi.org/10.1073/pnas.1508347112

Rouillard, A. D., Gundersen, G. W., Fernandez, N. F., Wang, Z., Monteiro, C. D., McDermott, M. G., \& Ma'ayan, A. (2016). The harmonizome: a collection of processed datasets gathered to serve and mine knowledge about genes and proteins. Database: The Journal of Biological Databases and Curation, 2016. https://doi.org/10.1093/database/baw100

Salas-Huetos, A., Blanco, J., Vidal, F., Grossmann, M., Pons, M. C., Garrido, N., \& Anton, E. (2016). Spermatozoa from normozoospermic fertile and infertile individuals convey a distinct miRNA cargo. Andrology, 4(6), 1028-1036.

https://doi.org/10.1111/andr.12276

Salas-Huetos, A., James, E. R., Aston, K. I., Carrell, D. T., Jenkins, T. G., \& Yeste, M. (2020). The role of miRNAs in male human reproduction: a systematic review. Andrology, Vol. 8, pp. 7-26. https://doi.org/10.1111/andr.12714

Sardiello, M., Licciulli, F., Catalano, D., Attimonelli, M., \& Caggese, C. (2003). MitoDrome: a database of Drosophila melanogaster nuclear genes encoding proteins targeted to the mitochondrion. Nucleic Acids Research, 31(1), 322. https://doi.org/10.1093/NAR/GKG123

Schagdarsurengin, U., \& Steger, K. (2016, October 1). Epigenetics in male reproduction: Effect of paternal diet on sperm quality and offspring health. Nature Reviews Urology, Vol. 13, pp. 584-595. https://doi.org/10.1038/nrurol.2016.157

Schindelin, J., Arganda-Carreras, I., Frise, E., Kaynig, V., Longair, M., Pietzsch, T., ... Cardona, A. (2012, June 28). Fiji: An open-source platform for biological-image analysis. Nature Methods, Vol. 9, pp. 676-682. https://doi.org/10.1038/nmeth.2019

Scialò, F., Fernández-Ayala, D. J., \& Sanz, A. (2017). Role of mitochondrial reverse electron transport in ROS signaling: Potential roles in health and disease. Frontiers in Physiology, Vol. 8. https://doi.org/10.3389/fphys.2017.00428

Sengupta, P., Dutta, S., \& Krajewska-Kulak, E. (2017). The Disappearing Sperms: Analysis of Reports Published Between 1980 and 2015. American Journal of Men's Health, 11(4), 1279-1304. https://doi.org/10.1177/1557988316643383

Sharma, U., Conine, C. C., Shea, J. M., Boskovic, A., Derr, A. G., Bing, X. Y., Belleannee, C., Kucukural, A., Serra, R. W., Sun, F., et al (2016). Biogenesis and function of tRNA fragments during sperm maturation and fertilization in mammals. Science, 351(6271), 391-396. https://doi.org/10.1126/science.aad6780

Sharma, U., Sun, F., Conine, C. C., Reichholf, B., Kukreja, S., Herzog, V. A., Ameres, S. L., \& Rando, O. J. (2018). Small RNAs Are Trafficked from the 
1201 Epididymis to Developing Mammalian Sperm. Developmental Cell, 46(4), 481 -

1202 494.e6. https://doi.org/10.1016/j.devcel.2018.06.023

1203 Skog, S., Örkenby, L., Kugelberg, U., Tariq, K., Farrants, A.-K. Ö., Öst, A., \& Nätt, D. (2021). Seqpac: A New Framework for small RNA analysis in R using SequenceBased Counts. BioRxiv, 2021.03.19.436151. https://doi.org/10.1101/2021.03.19.436151

1207 Stanger, S. J., Bernstein, I. R., Anderson, A. L., Hutcheon, K., Dun, M. D., Eamens, A. L., \& Nixon, B. (2020). The abundance of a transfer RNA-derived RNA fragment small RNA subpopulation is enriched in cauda spermatozoa. ExRNA, 2(1), 1-17. https://doi.org/10.1186/s41544-020-00058-x

Takemori, N., \& Yamamoto, M. T. (2009). Proteome mapping of the Drosophila melanogaster male reproductive system. Proteomics, 9(9), 2484-2493. https://doi.org/10.1002/pmic.200800795

Tavares, R. S., Escada-Rebelo, S., Correia, M., Mota, P. C., \& Ramalho-Santos, J. (2016). The non-genomic effects of endocrine-disrupting chemicals on mammalian sperm. Reproduction, Vol. 151, pp. R1-R13. https://doi.org/10.1530/REP-15-0355

Torra-Massana, M., Jodar, M., Barragán, M., Soler-Ventura, A., Delgado-Dueñas, D., Rodríguez, A., Oliva, R., \& Vassena, R. (2021). Altered mitochondrial function in spermatozoa from patients with repetitive fertilization failure after ICSI revealed by proteomics. Andrology, 9(4), 1192-1204. https://doi.org/10.1111/andr.12991

Tremellen, K. (2008). Oxidative stress and male infertility - A clinical perspective. Human Reproduction Update, Vol. 14, pp. 243-258.

https://doi.org/10.1093/humupd/dmn004

Trigg, N. A., Skerrett-Byrne, D. A., Xavier, M. J., Zhou, W., Anderson, A. L., Stanger, S. J., Katen, A. L., De luliis, G. N., Dun, M. D., et al (2021). Acrylamide modulates the mouse epididymal proteome to drive alterations in the sperm small non-coding RNA profile and dysregulate embryo development. Cell Reports, 37(1), 109787. https://doi.org/10.1016/j.celrep.2021.109787

Veal, E. A., Day, A. M., \& Morgan, B. A. (2007). Hydrogen Peroxide Sensing and Signaling. Molecular Cell, Vol. 26, pp. 1-14.

https://doi.org/10.1016/j.molcel.2007.03.016

Vojtech, L., Woo, S., Hughes, S., Levy, C., Ballweber, L., Sauteraud, R. P., Strobl, J., Westerberg, K., Gottardo, R., Tewari, M., \& Hladik, F. (2014). Exosomes in human semen carry a distinctive repertoire of small non-coding RNAs with potential regulatory functions. Nucleic acids research, 42(11), 7290-7304. https://doi.org/10.1093/nar/gku347

Wang, C., Yang, C., Chen, X., Yao, B., Yang, C., Zhu, C., Li, L., Wang, J., Li, X., Shao, Y., Liu, Y., et al (2011). Altered profile of seminal plasma microRNAs in the molecular diagnosis of male infertility. Clinical Chemistry, 57(12), 1722-1731. https://doi.org/10.1373/clinchem.2011.169714 
1241 Wang, J., Zhang, P., Lu, Y., Li, Y., Zheng, Y., Kan, Y., Chen, R., \& He, S. (2019). PiRBase: A comprehensive database of piRNA sequences. Nucleic Acids Research, 47(D1), D175-D180. https://doi.org/10.1093/nar/gky1043

Wasbrough, E. R., Dorus, S., Hester, S., Howard-Murkin, J., Lilley, K., Wilkin, E., Polpitiya, A., Petritis, K., \& Karr, T. L. (2010). The Drosophila melanogaster sperm proteome-II (DmSP-II). Journal of Proteomics, 73(11), 2171-2185. https://doi.org/10.1016/j.jprot.2010.09.002

Wickersheim, M. L., \& Blumenstiel, J. P. (2013). Terminator oligo blocking efficiently eliminates rRNA from Drosophila small RNA sequencing libraries. BioTechniques, 55(5), 269-272. https://doi.org/10.2144/000114102

Xu, H., Wang, X., Wang, Z., Li, J., Xu, Z., Miao, M., Chen, G., Lei, X., Wu, J., Shi, H., et al. (2020). MicroRNA expression profile analysis in sperm reveals hsa-mir-191 as an auspicious omen of in vitro fertilization. BMC Genomics, 21(1). https://doi.org/10.1186/s12864-020-6570-8 G., et al. (2020). Expression Pattern of Seminal Plasma Extracellular Vesicle Small RNAs in Boar Semen. Frontiers in veterinary science, 7, 585276. https://doi.org/10.3389/fvets.2020.585276

1259 Yuan, J., Zhang, P., Cui, Y., Wang, J., Skogerbø, G., Huang, D. W., Chen, R., \& He, 1260 S. (2016). Computational identification of piRNA targets on mouse mRNAs.

1261 Bioinformatics, 32(8), 1170-1177. https://doi.org/10.1093/bioinformatics/btv729

1262 Zhang, P., Kang, J. Y., Gou, L. T., Wang, J., Xue, Y., Skogerboe, G., Dai, P., Huang, 1263 D. W., Chen, R., et al (2015). MIWI and piRNA-mediated cleavage of messenger RNAs in mouse testes. Cell Research, 25(2), 193-207.

1265 https://doi.org/10.1038/cr.2015.4

1266 Zhang, P., Si, X., Skogerbø, G., Wang, J., Cui, D., Li, Y., Sun, X., Liu, L., Sun, B., 1267 Chen, R. et al (2014). PiRBase: A Web resource assisting piRNA functional study.

1268 Database, 2014. https://doi.org/10.1093/database/bau110

1269 Zhang, T., Sun, P., Geng, Q., Fan, H., Gong, Y., Hu, Y., Shan, L., Sun, Y., Shen, W., \& Zhou, Y. (2021). Disrupted spermatogenesis in a metabolic syndrome model: The role of vitamin A metabolism in the gut-testis axis. Gut. https://doi.org/10.1136/gutjnl- 
A

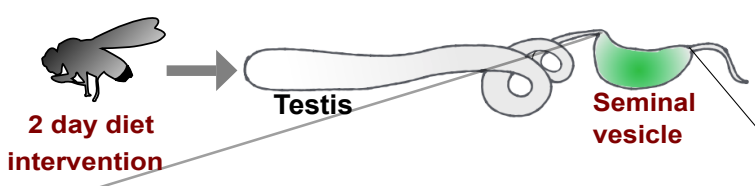

interver

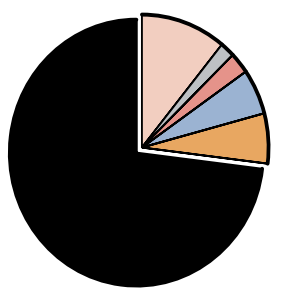

Total $=542$

C

GO:0006099 GO:0006091 GO:0015980 GO:0019752

GO:0043436

GO:0006082

GO:0055114

GO:0002181

GO:0043604

GO:0006412

GO:0043043

GO:0043603

GO:0044271

GO:1901566

GO enrichment: Cluster 1

- Metabolic proteins

Structural proteins

ㄷsps

acps

athers

Go enrichment: Cluster 2

Cytoplasmic translation

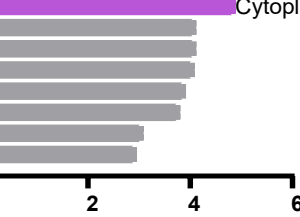

$\log _{2}$ Fold change

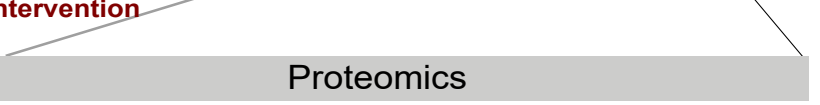

च Translation/Ribosome

B Z score

$\begin{array}{llll}-2 & 0 & 2\end{array}$

$3 \mathrm{~g} / \mathrm{L}$ $30 \mathrm{~g} / \mathrm{L}$ $300 \mathrm{~g} / \mathrm{L}$

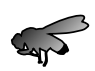

11

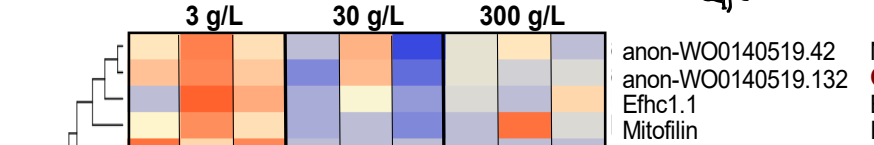

Not known

GAPDH

IMMT, Mic60

ALDH3B1

ATP1A1

ME1,ME,ME3

GFM2

CYLC1

PKM

CHI3L1
MYL2

MYL2

CS

Vha68-3

anon-WO0140519.178

UGP2

UGP

Fum2

kl-5

Rsph4a

CG8349

CG5265

Pp1-13C

CG32026

RpL8

GstS1

Obp51a

0.023
0.030

$\begin{array}{rr}\text { Pyruvate metabolic process } & 0.030 \\ 0.001 \\ 0.037 \\ 0.014\end{array}$

0.014
0.012

0.010

0.042

0.008

0.017

0.017
0.0005

0.0005
0.002

0.002

0.0008

0.0005

0.015
0.025

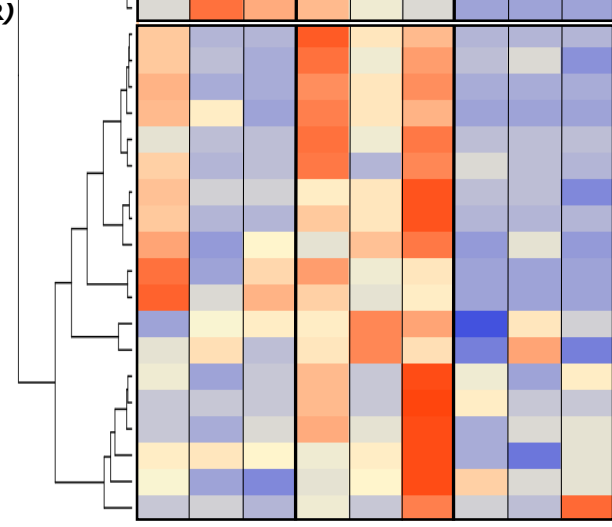

NLaz

Acp53C14b

Ter94

RpL22-like

CG34034

$\mathrm{RpL} 7$

Irp-1B

glob1

glob1

CG5162

CCT4

elF4A

EF2

Jupiter

$\mathrm{FH}$

DNAH9

TKT,TKTL2

RSPH4A

CDA

CRAT

PPP1CA

IDH3A

RPL8

HPGDS

Not known

APOD

$\mathrm{CHDH}$

Not known

VCP

RPL22,RPL22L

Not known

RPL7

ACO1

CYGB

DLAT

LIPC

CCT4

EIF4A1

EEF2

JPT2

EF1A2 
bioRxiv preprint doi: https://doi.org/10.1101/2021.10.20.465156; this version posted October 20, 2021. The copyright holder for this preprint (which was not certified by peer review) is the author/funder, who has granted bioRxiv a license to display the preprint in perpetuity. It is made available under aCC-BY-NC-ND 4.0 International license.

\section{Figure 2}
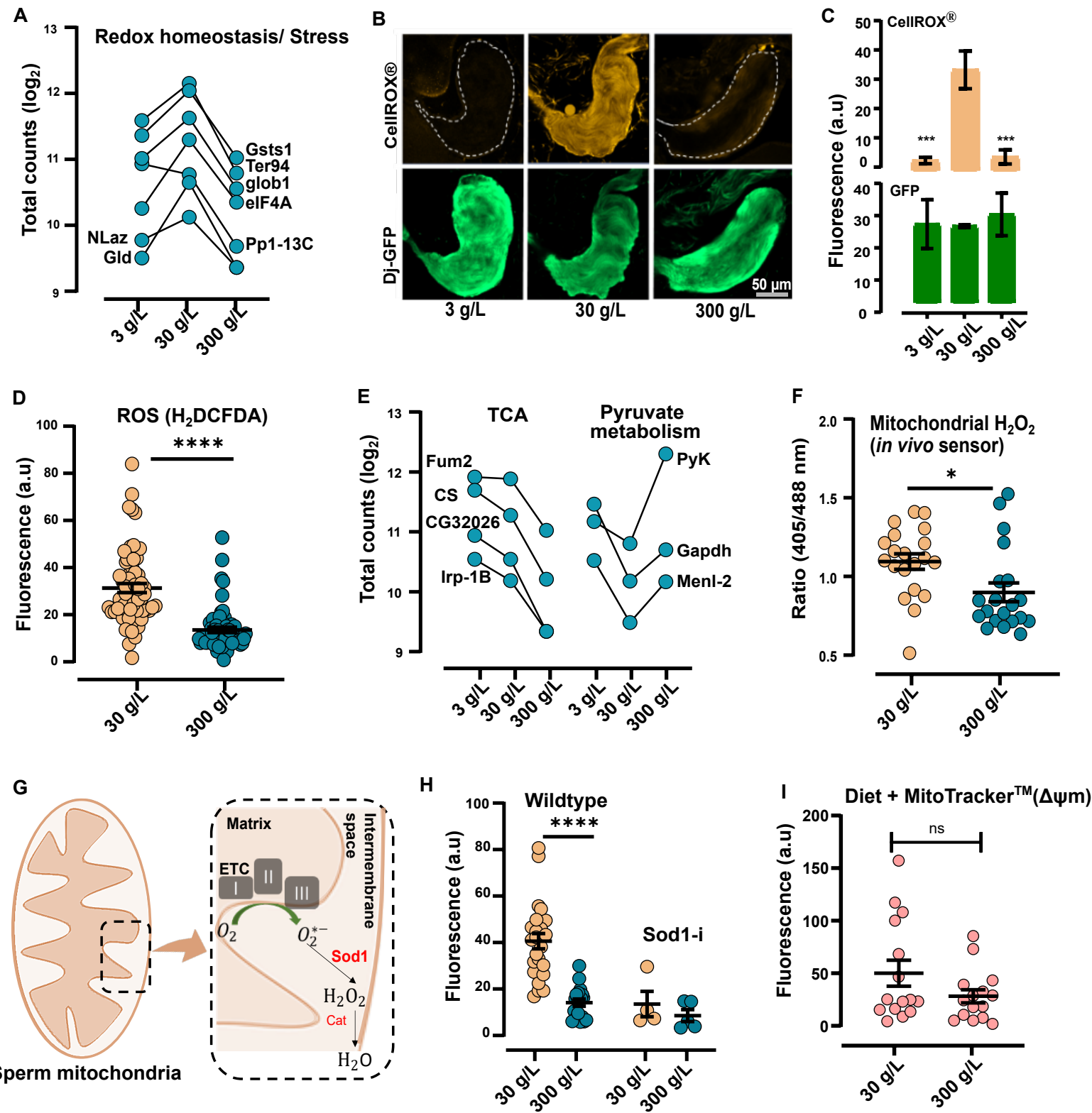
bioRxiv preprint doi: https://doi.org/10.1101/2021.10.20.465156; this version posted October 20, 2021. The copyright holder for this preprint (which was not certified by peer review) is the author/funder, who has granted bioRxiv a license to display the preprint in perpetuity. It is made available under aCC-BY-NC-ND 4.0 International license.

\section{Figure 3}

A

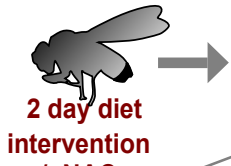

+ + NAC

Diet and NAC associated ROS in seminal vesicles

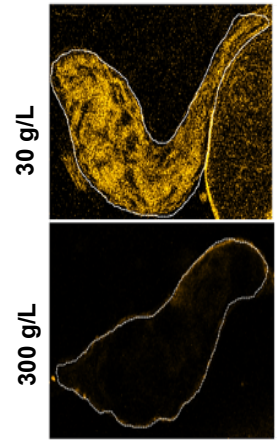

- NAC

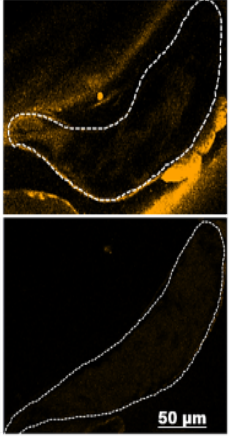

+ NAC

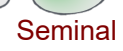

Mature sperm
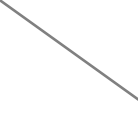

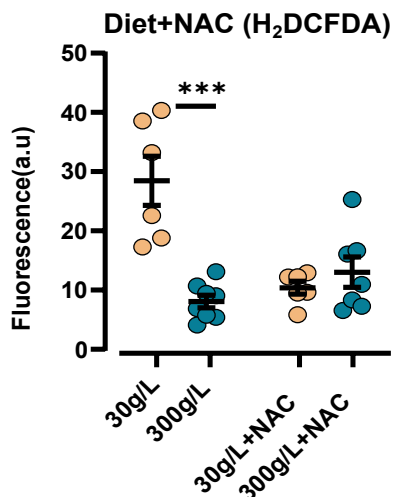

B vesicle

Diet and NAC associated SRNA in mature sperm

C

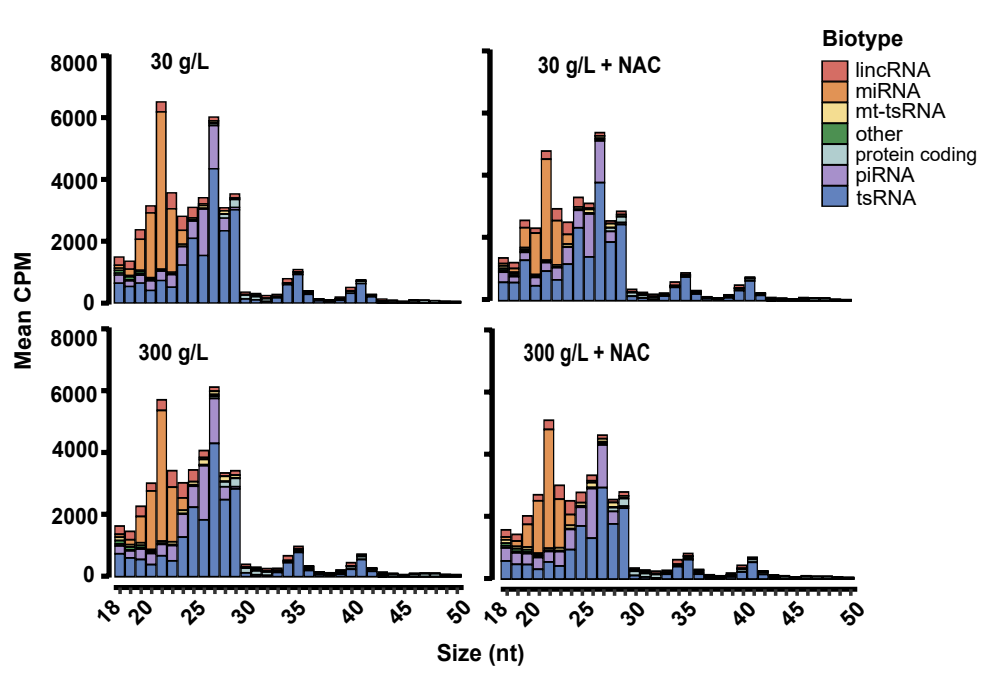

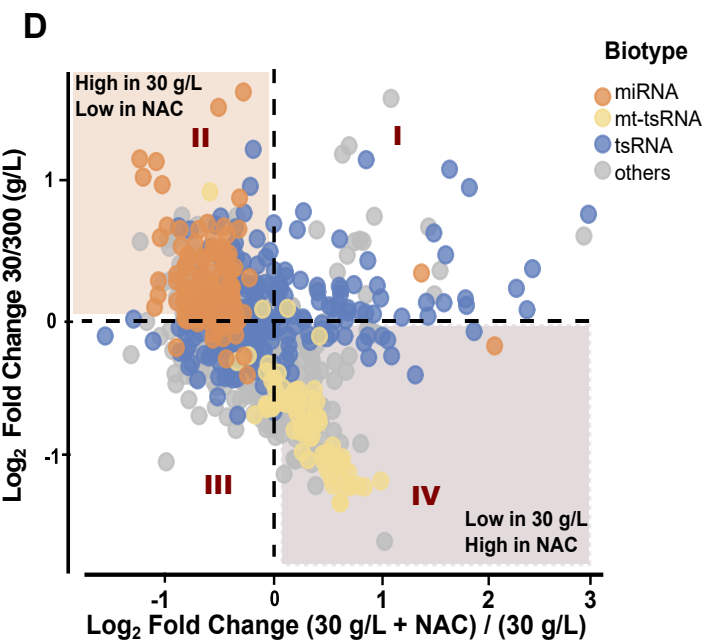

$\log _{2}$ Fold Change (30 g/L + NAC) / (30 g/L) miRNA changes in mature sperm

E

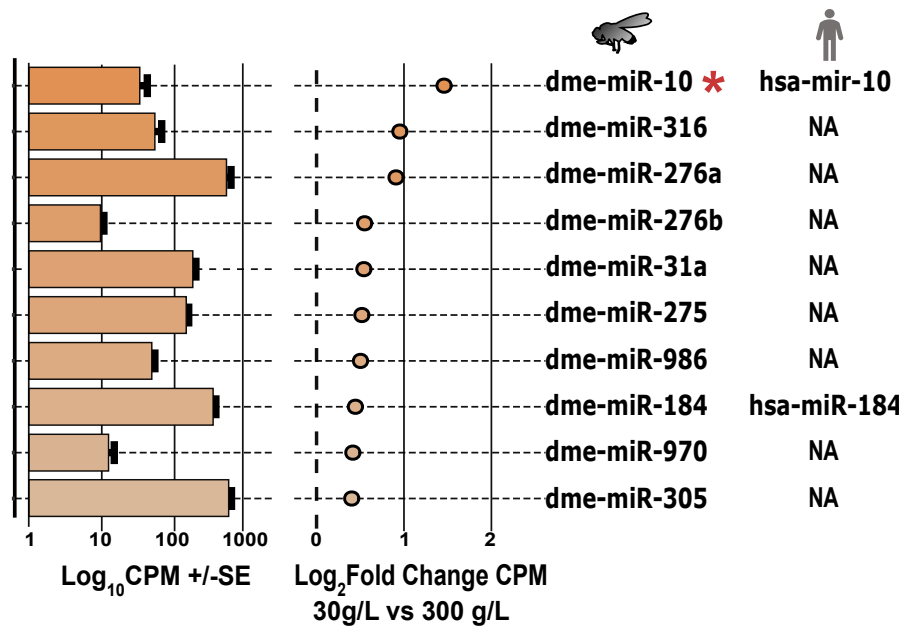

F dme-miR-10

G Coverage plot dme-miR-10
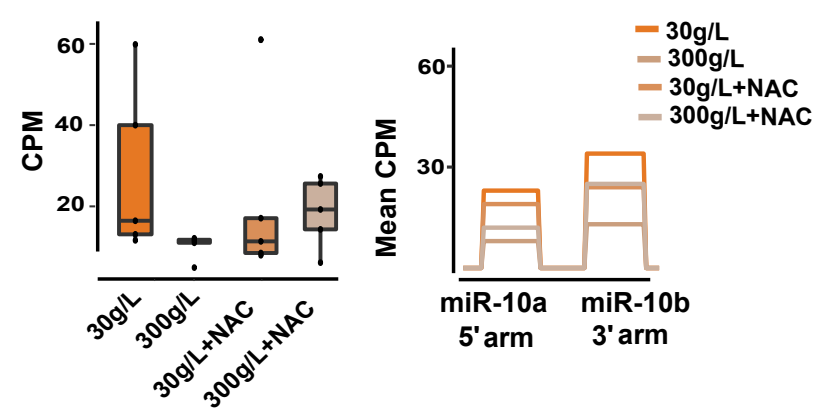
bioRxiv preprint doi: https://doi. org/10.1101/2021.10.20.465156; this version posted October 20, 2021. The copyright holder for this preprint (which was not certified by peer review) is the author/funder, who has granted bioRxiv a license to display the preprint in perpetuity. It is made available under aCC-BY-NC-ND 4.0 International license.

\section{Figure 4}

\section{A tsRNA classification}

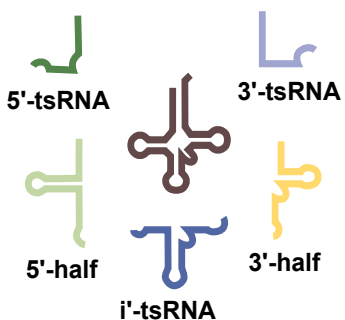

B Distribution

Mitochondrial tsRNA

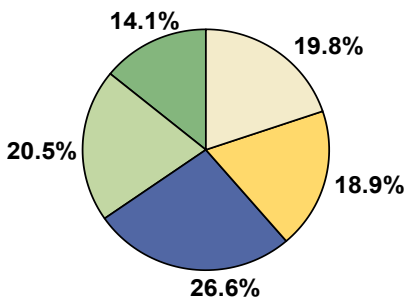

Nuclear tsRNA

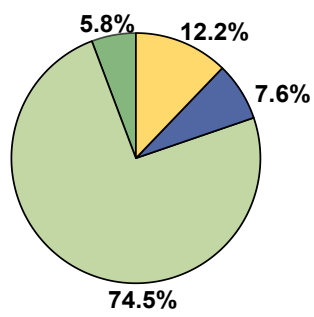

\section{Diet and NAC effects on tsRNA}

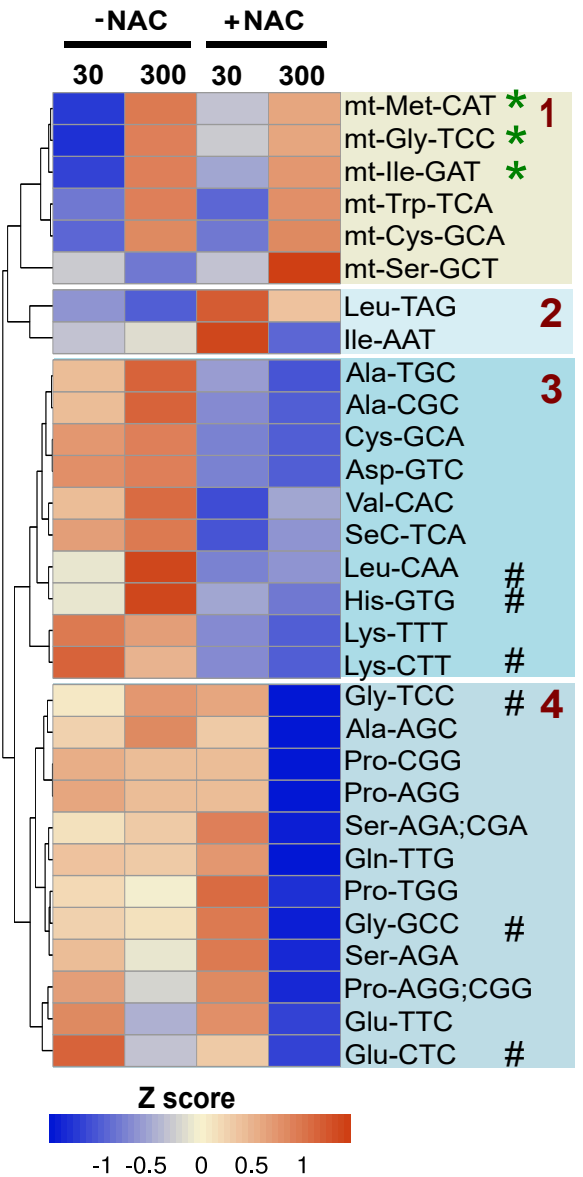

D Diet-sensitive mt-tsRNA, $p<0.05$

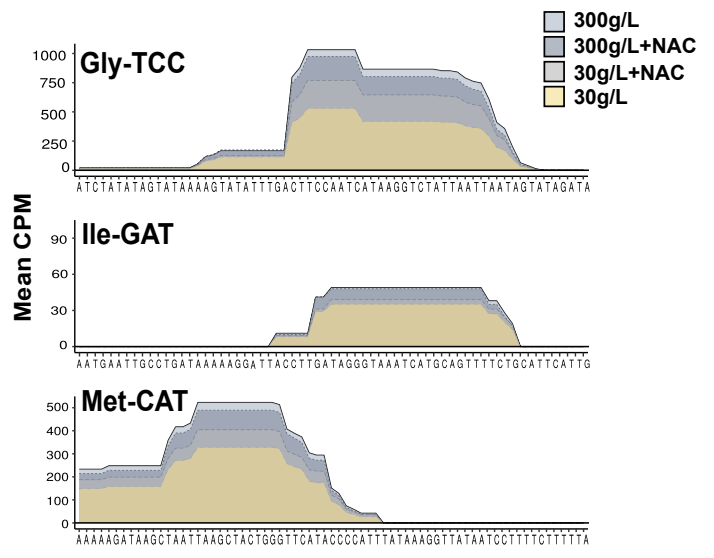

E Diet-sensitivity of mt-tsRNA across species
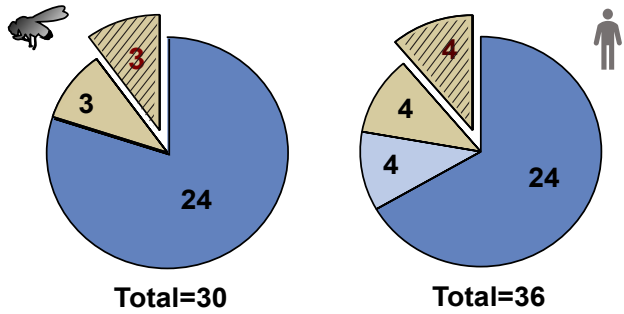

nuclear tsRNA

$p<0.05$, nuclear tsRNA

$\square$ mt-tsRNA

$p<0.05$, mt-tsRNA 


\section{Supplementary Figure 1}

A

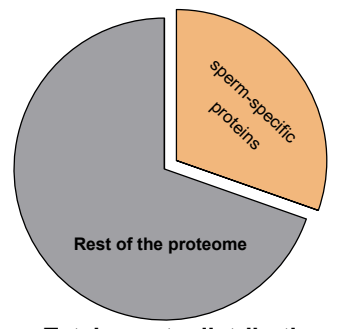

Total counts distribution
B

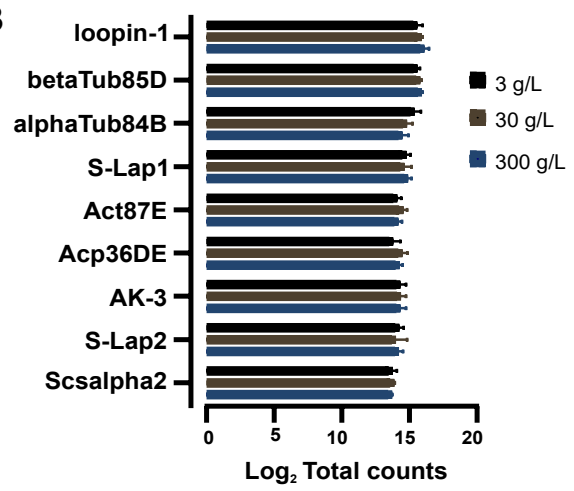

D Testes phenotype after RNAi

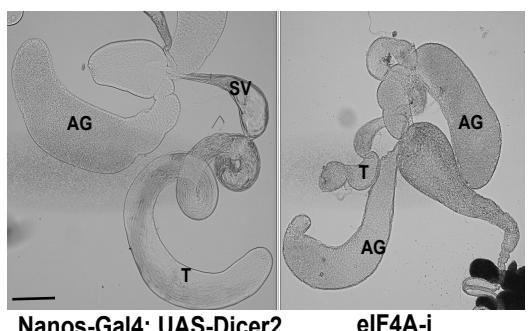

Nanos-Gal4; UAS-Dicer2

\section{RNAi scheme}

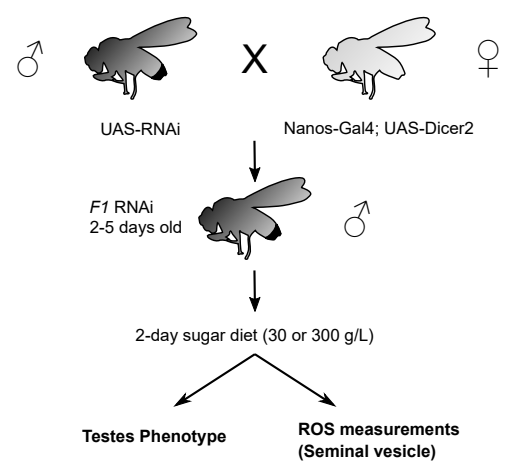


bioRxiv preprint doi: https://doi.org/10.1101/2021.10 20.465156: this version posted October 20, 2021. The copyright holder for this

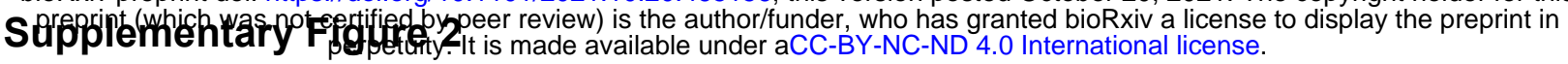

A mito-roGFP2-Orp1 is expressed and can be visualised in sperm

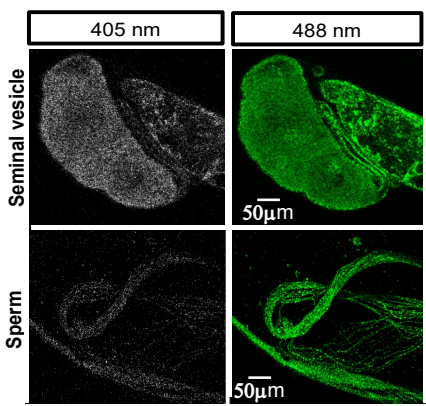

C Redox status after dietary intervention

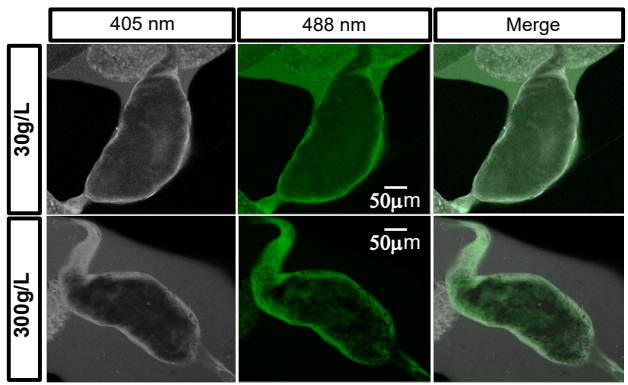

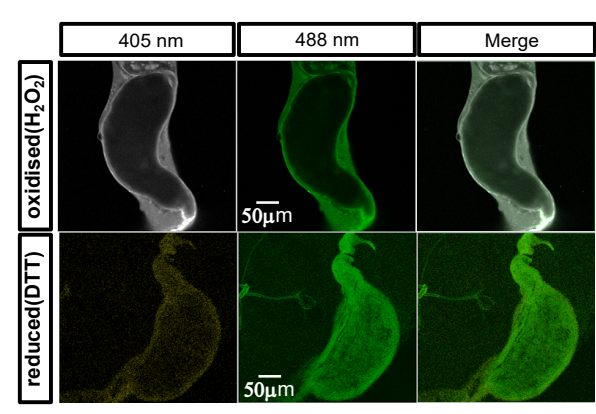

B Establishment of dynamic range

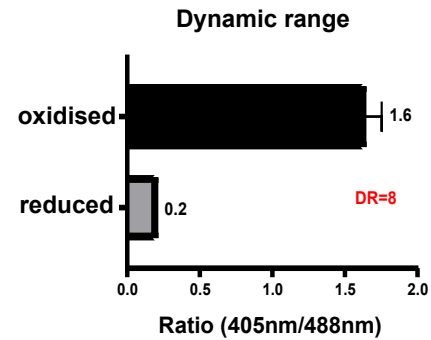

D Measurement of mitochondrial potential

E Quantification of ROS ( $\mathrm{H}_{2}$ DCFDA)

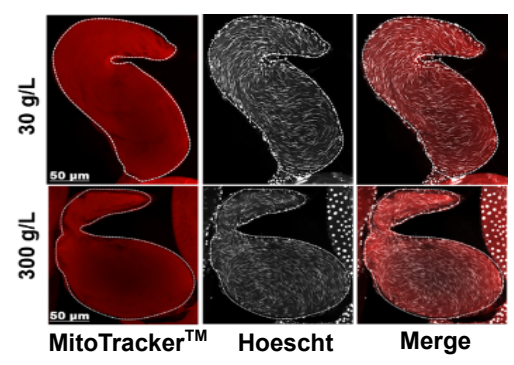




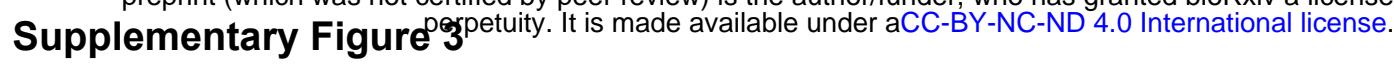

\section{A Dietary effects on sRNA}

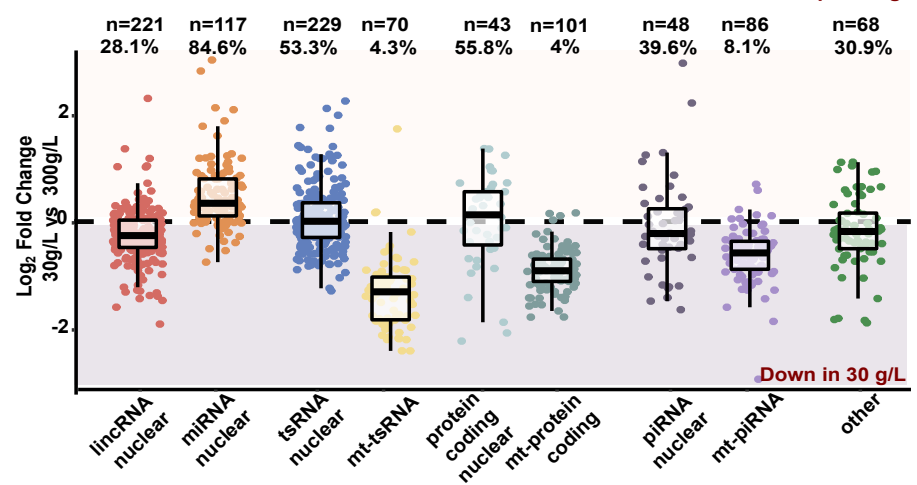

B NAC effects in $30 \mathrm{~g} / \mathrm{L}$ diet
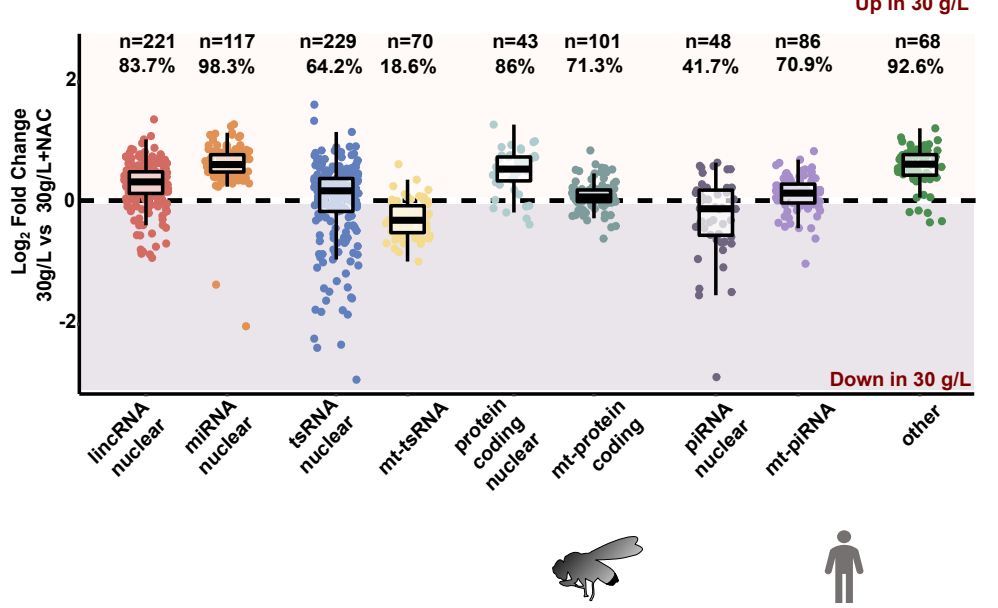

C Diffferential expression of miRNA between diets

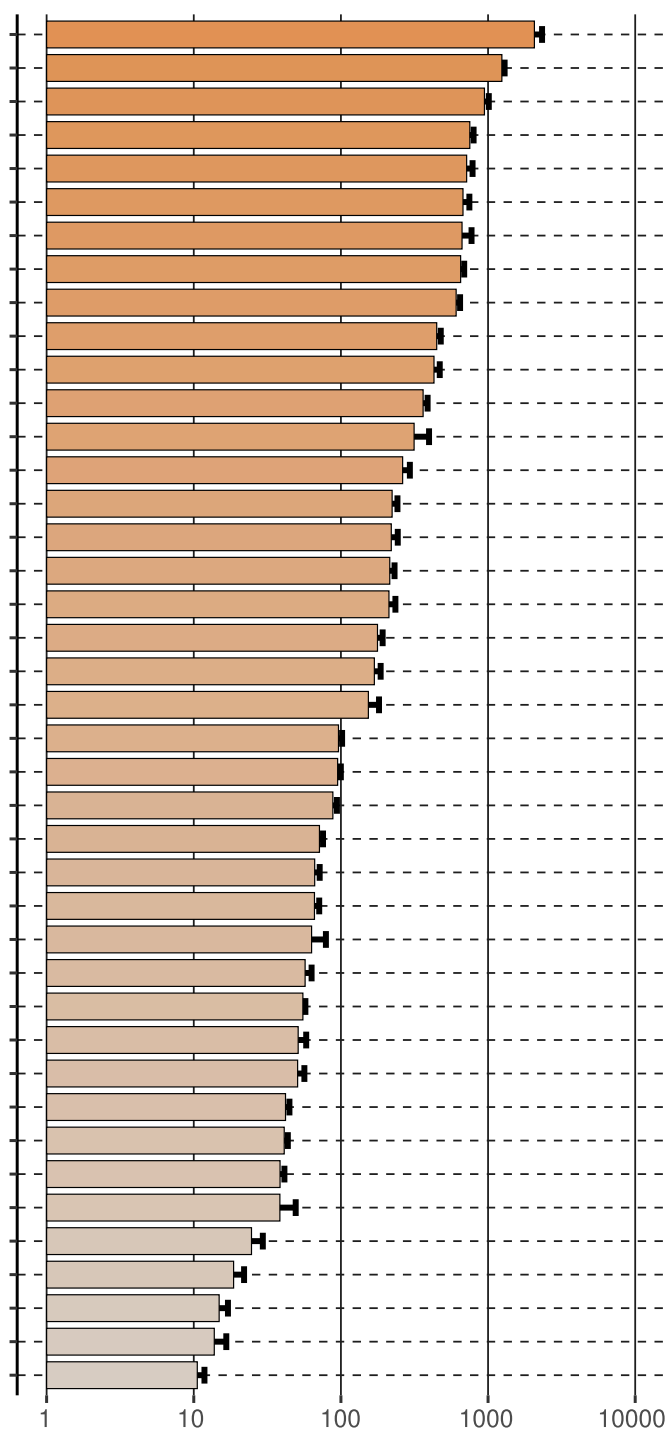

$\log _{10} \mathrm{CPM}+/-\mathrm{SE}$

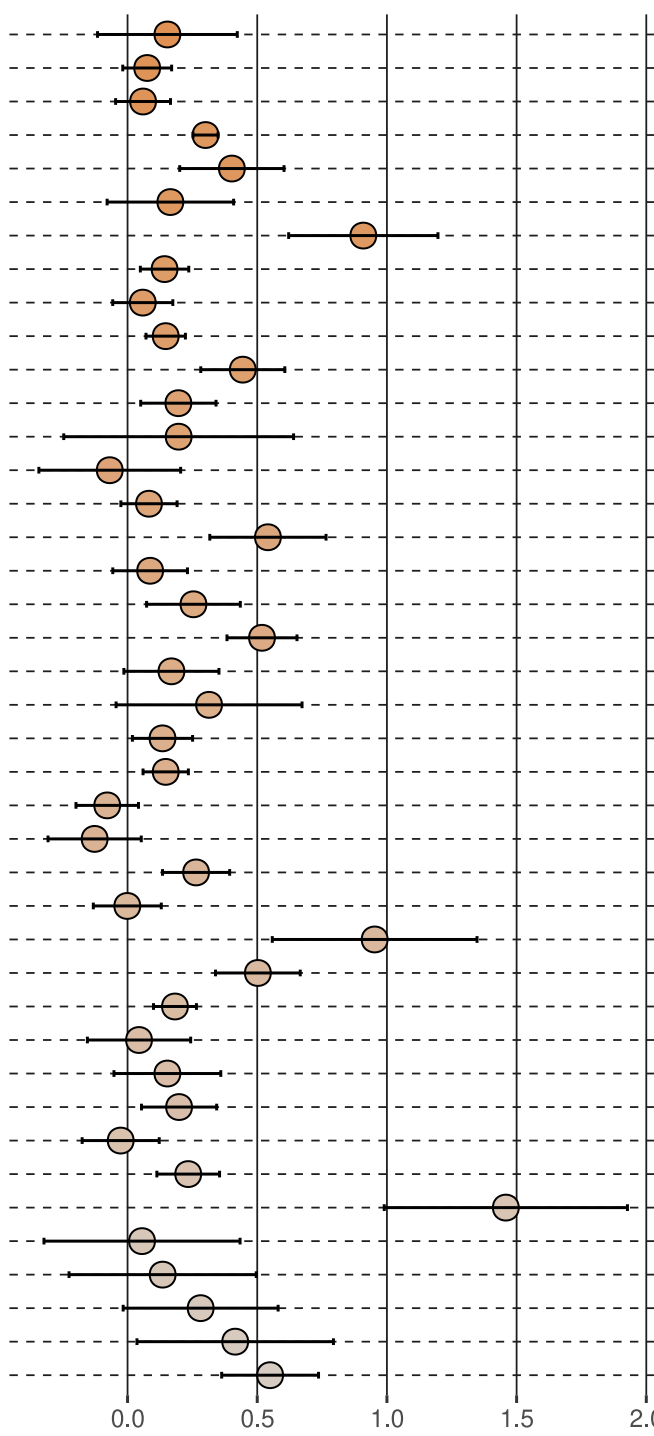

dme-miR-1 dme-miR-9a dme-miR-8 dme-bantam dme-miR-305 dme-miR-14 dme-miR-276a dme-miR-34

dme-let-7 dme-miR-11 dme-miR-184 dme-miR-7

dme-miR-375 dme-miR-263a

dme-miR-279

dme-miR-31a

dme-miR-278

dme-miR-277

dme-miR-275

dme-miR-999

dme-miR-283

dme-miR-9b

dme-miR-317

dme-miR-12

dme-miR-284

dme-miR-33

dme-miR-31b

dme-miR-316

dme-miR-986

dme-miR-100

dme-miR-988

dme-miR-965

dme-miR-125

dme-miR-304

dme-miR-9c

dme-miR-10

dme-miR-281-1

dme-miR-1012

dme-miR-996

dme-miR-970

dme-miR-276b
hsa-miR-1/206

NA

hsa-miR-200

NA

NA

NA

hsa-miR-34

hsa-let-7

NA

hsa-miR-184

hsa-miR-7

hsa-miR-375

NA

NA

NA

NA

NA

NA

NA

NA

NA

NA

NA

NA

hsa-miR-33

NA

NA

NA

NA

NA

NA

NA

NA

hsa-miR-10 *

NA

NA

NA

NA

NA log2 Fold Change +/- SE 


\section{Supplementary Figure 4}

A

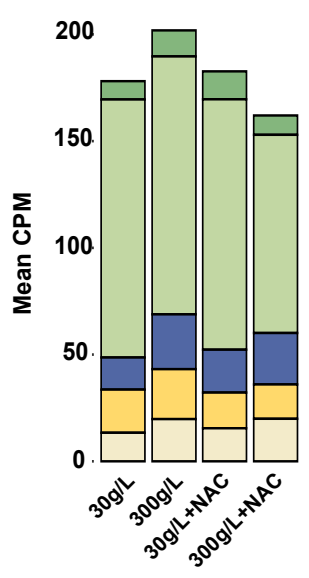

B

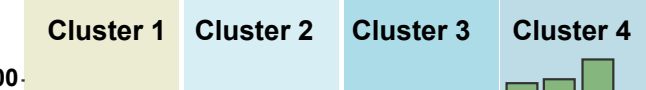

200.

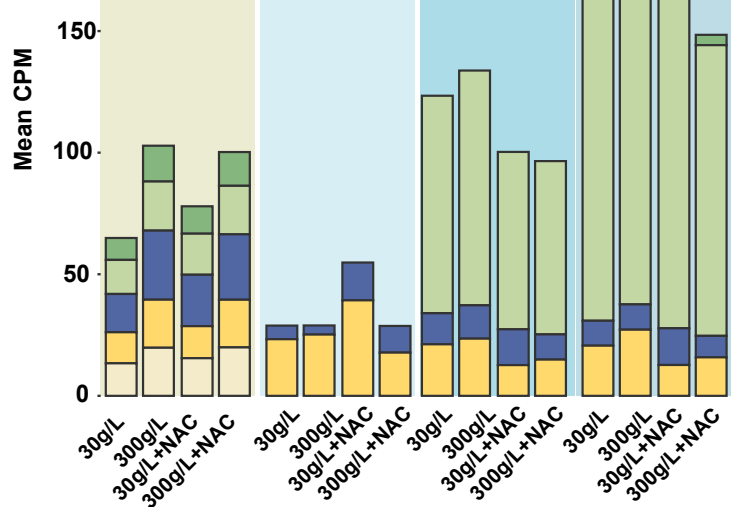

C

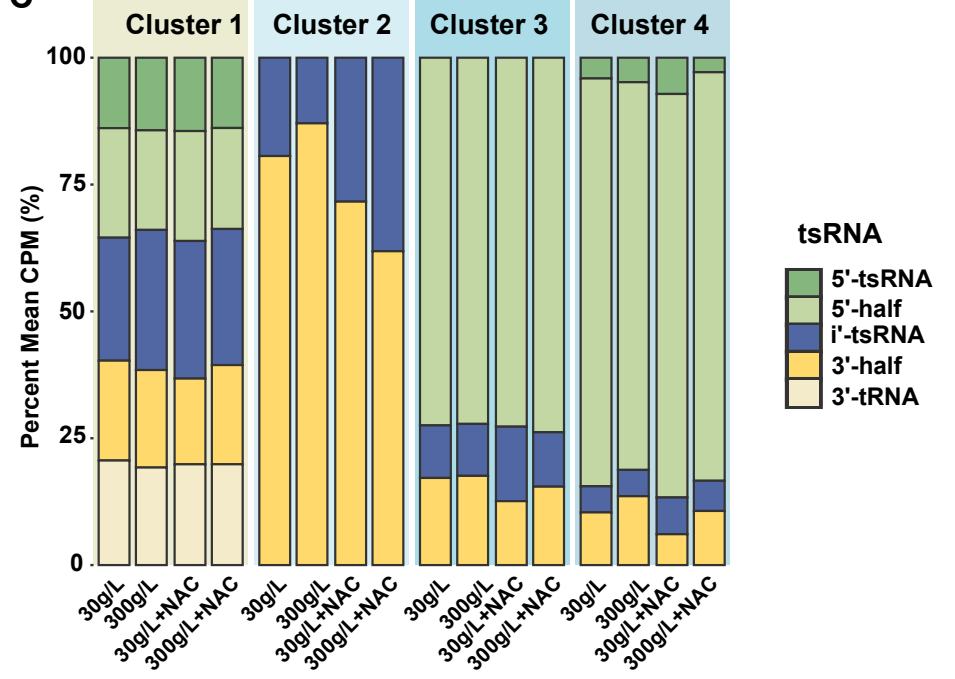

